\title{
Relation between the guessed and the derived super-Hamiltonians for spherically symmetric shells
}

\author{
Petr Hájíček \\ Institute for Theoretical Physics, University of Bern, Sidlerstrasse 5, CH-3012 Bern, Switzerland
}

(April 1998)

\begin{abstract}
The Hamiltonian dynamics of spherically symmetric massive thin shells in the general relativity is studied. Two different constraint dynamical systems representing this dynamics have been described recently; the relation of these two systems is investigated. The symmetry groups of both systems are found. New variables are used, which among other things simplify the complicated system a great deal. The systems are reduced to presymplectic manifolds $\Gamma_{1}$ and $\Gamma_{2}$, lest non-physical aspects like gauge fixings or embeddings in extended phase spaces complicate the line of reasoning. The following facts are shown. $\Gamma_{1}$ is three- and $\Gamma_{2}$ is five-dimensional; the description of the shell dynamics by $\Gamma_{1}$ is incomplete so that some measurable properties of the shell cannot be predicted. $\Gamma_{1}$ is locally equivalent to a subsystem of $\Gamma_{2}$ and the corresponding local morphisms are not unique, due to the large symmetry group of $\Gamma_{2}$. Some consequences for the recent extensions of the quantum shell dynamics through the singularity are discussed.
\end{abstract}

04.60.Ds, 04.20.Fy 


\section{INTRODUCTION}

The dominating physical problem of the theory of gravitation is the gravitational collapse and the inevitable singularity. That is the point, where the classical theory breaks down; one expects that the quantum theory will help.

The project of which the present paper is a part focuses on simplified models of gravitating systems, whose quantum mechnics can be constructed without much technical and conceptual difficulty. We hope that such models can help us to find quantization methods that are, in short, a) gauge (reparametrization) invariant and b) liberated from semiclassical thinking. Indeed, the gauge invariance is an issue, because a gauge fixation leads much more easily to gauge-dependent results in quantum gravity than, say, in the Yang-Mills field theory. This has to do with the so-called problem of time [1], [2]. The term 'semi-classical thinking' is to express uneasiness about the extensions to the gravidynamics of the naive assumption that every dynamics is a theory of motion of some objects in some spacetime (cf. 'covariant quantization' [3], string theory [4], or 'effective field theory' [5]). As the spacetime is itself dynamical in the presence of gravitation, the only method complying with the assumption seems to be an expansion around a classical solution, and so it must be, it seems at least, a sort of WKB approximation.

Our pet model is the spherically symmetric thin shell of dust in the general relativity. In refs. [6], [7], [8] and [9], a sufficiently simple super-Hamiltonian for this system was guessed from the equations of motion, so that the model could be readily quantized. We shall call this method the Warsaw approach円. This quantum theory lead to some unexpected results:

1. Existence of a unitary scattering theory; after a contracting phase, the shell goes through a more or less probable intermediate state of black and white hole and then spacetime wave packet expands again; the probability of the hole stage depends on the

\footnotetext{
${ }^{1}$ A systematic and general exposition of this method (Ref. [9]) was first given at a Banach Center Workshop, Warsaw.
} 
energy of the shell.

2. This unitary regime exists for all values of energy but only if the total rest mass does not exceed the Planck mass; what happens with more massive shells remains unclear.

3. There is no spacetime geometry that can be associated with any particular scattering process: the wave packets contain linear combinations of wave functions that describe spacetimes with contracting shells and black holes as well as spacetimes with expanding shells and a white holes.

The method, however, has several weak points.

1. The way from a dynamical equation to a Hamiltonian principle is non-unique and it is unclear how much the final quantum results depend on a particular guess. Some kind of uniqueness has been shown in Ref. [9], but this is not yet completely reassuring, as we shall also see in the present paper.

2. The great simplicity of the super-Hamiltonian is achieved by a very special choice of variables: they are the coordinates of the shell in the subspacetime at one side of the shell. This subspacetime of description (left subspacetime) lies on the opposite side of the shell than the subspacetime where the observers spend most of their time (right subspacetime). The drawback is that some observable properties of the shell are not contained among (or calculable from) these variables. A controversy arises as to whether and where the observers will see the expanding shell. Undetermined are for example the scattering time delays of the expanding packet with respect to the contracting one (usually given by the derivatives with respect to energy of the phase shifts of the S-matrix), which should in principle be measurable. The possibility was even discussed that the wave packet expanded into a different right subspacetime than where it originally contracted so that the scattering time delays made no sense.

3. The method of self-adjoint extension of Hamiltonian operator was applied in Refs. [6], [7], and [8] to make the quantum evolution complete (unitary). This method seemed 
to some physicists too formal and suspicious to cope satisfactorily with the problem of the singularity.

The present paper is an attempt to deal with all three problems. The analysis will be based on Refs. [10] and [11]; we shall refer to this approach as the Potsdam one ${ }^{2}$. The Potsdam Hamiltonian action for spherically symmetric massive shells has been derived from first principles, so one can say something about the first problem. The Postdam dynamics of the shell is described by its coordinates in both subspacetimes left and right from the shell, and so it contains complete information about the motion. This enables us to say something about the second problem. Finally, we shall find that the method of self-adjoint extension is incomplete in certain respect, and so there is some information about the third problem, too. There is, of course, a strong motivation for the Warsaw approach: this is the etremely simple form of its action.

The plan of the paper is as follows. In Sec. III, we shall briefly collect the results of Refs. [9] and [11] that will be needed, so that the paper becomes self-contained. New is the systematic use of the Kruskal coordinates and more or less complete description of the symmetries that the systems admit. Sec. III is based on the idea that the 'naked' or minimal mathematical structure that underlies a given constrained system is the constraint manifold together with the induced presymplectic form. According to this idea, two constrained systems are equivalent, if they define the same presymplectic manifolds. This notion is completely gauge (and reparametrization) invariant; it is moreover independent of the details of imbedding of the constraint manifold in an extended phase space, which is not physical and not unique. In Sec. III, we reduce the Warsaw and the Potsdam descriptions to these 'naked' forms. The Warsaw presymplectic manifold $\left(\Gamma_{1}, \Theta_{1}\right)$ is three-dimensional and the Potsdam presymplectic manifold $\left(\Gamma_{2}, \Theta_{2}\right)$ is five-dimensional. As a byproduct of the transformations made in this section, 'miraculous' new variables are found which considerably simplify the

\footnotetext{
${ }^{2}$ Ref. [11] was written down at the Albert Einstein Institute, Potsdam.
} 
Potsdam action.

In Sec. IV], we pick up a three-dimensional subsystem $\left(\Gamma_{E}, \Theta_{E}\right)$ from the Potsdam system $\left(\Gamma_{2}, \Theta_{2}\right)$ that has a chance to be equivalent to a particular Warsaw system. Concretely, the Schwarzschild mass of the left subspacetime must be fixed, and the corresponding cyclic coordinate must be excluded. Sec. $\nabla$ is devoted to the search of a morphism of presymplectic manifolds that would map $\left(\Gamma_{1}, \Theta_{1}\right)$ onto $\left(\Gamma_{E}, \Theta_{E}\right)$. We set up a partial differential equation for this map and solve it in suitable coordinates. A careful study of the results reveals that

1. the two presymplectic manifolds are locally but not globally equivalent, and

2. the local equivalence maps (morphisms of presymplectic manifolds) are not unique.

In more detail, the manifolds $\Gamma_{1}$ and $\Gamma_{E}$ can be covered with open patches such that each one on $\Gamma_{1}$ is equivalent to one on $\Gamma_{E}$, but the map cannot be extended to the outside of the patch, because it diverges at the boundary. Scattering trajectories contracting from right (left) and expanding to right (left) can both never lie within one and the same patch. Where two patches overlap, the two corresponding maps differ by a symmetry. The huge amount of symmetry in the Potsdam approach is the cause for the non-uniqueness of the map.

Let us dwell a little more on these results. The local equivalence explains why the same radial equation results from both systems. What is, however, a possible physical significance of just local but not global equivalence of constraint dynamical systems remains unclear. On could imagine, for example, pasting together several copies of $\Gamma_{1}$ and $\Gamma_{E}$ using the local equivalence maps. The result, however, seems to be a (possibly non-Hausdorff) presymplectic manifold with no reasonable physical interpretation (this is discussed at the end of Sec. $\nabla C$ ).

It is also conceivable that the global inequivalence of the systems is not important for the quantum theory; the delicate points where the map can diverge, lie all at the boundary between the scattering and the bound trajectories. One can speculate that this discontinuity is not relevant for the quantum mechanics, because the bound states become discrete anyway. 
If we cut out this less dimensional boundary from both classical systems, we obtain systems that are globally equivalent. Further study is necessary.

Suppose next that some form of weakened equivalence between the two systems makes sense. Then, given an equivalence map, we can consider the Warsaw system as a part of the Potsdam one, and the missing information about the position of the shell in the right subspacetime can be provided. The Warsaw variables can be regarded as coordinates on a part of the Potsdam system. In fact, it turns out then that the Warsaw variables are coordinates of the shell in the left subspacetime only according to their name; in reality, they play the role of coordinates in the right subspacetime. Hence, the position of the shell in the right subspacetime is well-determined; paradoxically, it is the position of the shell in the left subspacetime that is uncertain (see the discussion at the beginning of Sec. $\mathrm{V}$ ).

Applying this point of view to the quantum mechanics of Refs. [6] and [7], we can make some progress. The self-adjoint extension of the Hamiltonian defines a unitary dynamics in the Warsaw coordinates of a part of the Potsdam system. It follows that each wave packet reemerges, during the expanding part of this unitary evolution, in the same right subspacetime in which it originally started to contract, because the variables describing it all the time are coordinates on this subspacetime. This is discussed in more detail at the beginning of Sec. $\mathrm{V}$.

On the other hand, the non-uniqueness of the equivalence map has an unpleasant consequence: although the self-adjoint extension of the dynamics seems to be unique, it is so only with respect to a particular choice of Warsaw variables. Different equivalence maps lead to different choices of the variables and these, in turn, to different dynamics of the Potsdam system. This is explained at the beginning of Sec. $\nabla$. The difference is measurable, because the resulting scattering time delays are different. This suggests that one either has to look for some additional principle that could, together with the self-adjoint extension, lead to a unique set of time delays, or to look for some interpretation of the non-uniqueness (like, say, a loss of information) or to look for another way of dealing with the singularity. Future research may clarify the point. 


\section{DESCRIPTION OF THE SHELL DYNAMICS}

In this section, we briefly collect and round off some results scattered in literature making so the paper self-contained.

\section{A. The shell spacetime}

A sherically symmetric thin-shell spacetime solution of Einstein equations will be described in this subsection following closely Ref. [9]. Consider two Kruskal spacetimes $\mathcal{M}_{1}$ and $\mathcal{M}_{2}$ with Schwarzschild masses $E_{1}$ and $E_{2}$. Let $\Sigma_{1}$ be a timelike hypersurface in $\mathcal{M}_{1}$ and $\Sigma_{2}$ be one in $\mathcal{M}_{2}$. Let $\Sigma_{1}$ divides $\mathcal{M}_{1}$ into two subspacetimes, $\mathcal{M}_{1+}$ and $\mathcal{M}_{1-}$, and similarly $\Sigma_{2}$ divides $\mathcal{M}_{2}$ into $\mathcal{M}_{2+}$ and $\mathcal{M}_{2-}$; we chose fixed time and space orientation in the two-dimensional Kruskal spacetimes so that future and past as well as right and left are unambiguous; let then $\mathcal{M}_{2+}$ and $\mathcal{M}_{1+}$ be right with respect to $\mathcal{M}_{2-}$ and $\mathcal{M}_{1-}$. Let $\Sigma_{1}$ and $\Sigma_{2}$ be isometric; then the spacetime $\mathcal{M}_{1-}$ can be pasted together with the spacetime $\mathcal{M}_{2+}$ along the boundaries $\Sigma_{1}$ and $\Sigma_{2}$. The result is a shell spacetime $\mathcal{M}_{s}$. As everything is spherically symmetric, only the two-dimensional Kruskal spacetimes are relevant.

The observers are assumed to be in the asymptotic region of $\mathcal{M}_{2+}$. Given a shell spacetime, we shall often leave out the indices 1 and 2 , having right (left) energy $E_{+}\left(E_{-}\right)$, shell trajectory $\Sigma$, and the right (left) subspacetime $\mathcal{M}_{+}\left(\mathcal{M}_{-}\right)$. Thus, $\mathcal{M}_{+}=\mathcal{M}_{2} \cap \mathcal{M}_{s}$ and $\mathcal{M}_{-}=\mathcal{M}_{1} \cap \mathcal{M}_{s}$

The three-dimensional shell surface $\Sigma$ carries the energy-momentum tensor $T^{k l}$ of the shell. This will be assumed in the form of ideal fluid

$$
T^{k l}=(\rho+p) T^{k} T^{l}+p \gamma^{k l}
$$

where $\rho$ is the surface mass density, $p$ the negative surface tension, $T^{k}$ a unite timelike vector

field (the three-velocity of the fluid) tangential to $\Sigma$, and $\gamma_{k l}$ is the metric induced on the shell from the surrounding spacetime. Let $p=p(\rho)$ be the equation of state. 
The spherical symmetry and the energy-momentum conservation lead to the matter equation

$$
\mathcal{A} \frac{d \rho}{d \mathcal{A}}+\rho+p(\rho)=0
$$

where $\mathcal{A}$ is the surface of the shell, $\mathcal{A}:=4 \pi R^{2}$. We choose one of the particular solutions of the matter equation, $\rho(\mathcal{A})$, and define the so-called mass function $M(R)$ by

$$
M(R):=\mathcal{A}(R) \rho(\mathcal{A}(R))
$$

For example, the dust equation of state, $p=0$, implies that $M(R)=$ const, and each value of the constant defines a particular solution.

The spacetime around the shell already satisfies the Einstein equations; thus, the only non-trivial equation still to be satisfied is the jump condition, the so-called Israel equation [12]. It implies (for a derivation, see Ref. [9]) the following two equations for the embedding functions $T_{\epsilon}(s)$ and $R(s)$ of the shell in $\mathcal{M}_{\epsilon}$, where $\epsilon$ is a sign, $\epsilon= \pm 1$ and $s$ is the proper time along the radial generators of the surface.

A) The radial equation:

$$
\dot{R}^{2}+V(R)=0
$$

where

$$
V(R):=-\frac{M^{2}(R)}{4 R^{2}}-\frac{E_{+}+E_{-}}{R}-\frac{\left(E_{+}-E_{-}\right)^{2}}{M^{2}(R)}+1,
$$

and

B) the time equation (valid for all future oriented shell motions)

$$
\dot{T}_{\epsilon}=\frac{1}{M(R) F_{\epsilon}}\left(E_{+}-E_{-}-\epsilon \frac{M^{2}(R)}{2 R}\right),
$$

where

$$
F_{\epsilon}:=1-\frac{2 E_{\epsilon}}{R}
$$


is the Schwarzschild function.

Here, $T_{ \pm}$and $R$ are the Schwarzschild coordinates of the shell in $\mathcal{M}_{ \pm}$(they are of course singular at the four horizons of the two spacetimes $\mathcal{M}_{ \pm}$)

There are two types of solutions: bound and scattering; the scattering trajectories can be divided into expanding $(\dot{R}>0)$ to the right and to the left, and contracting $(\dot{R}<0)$ from the right and from the left (the usual notions of out- and in-going are not adequate to dynamics in the Kruskal spacetime). We stress that the four possibilities are unambiguous (see Ref. [9]): for each (scattering) shell spacetime, only one of these is realized in both subspacetimes $\mathcal{M}_{ \pm}$simultaneously.

\section{B. Warsaw approach}

In this subsection, we describe a short-cut approach (cf. e.g. [7] and [9]) to the shell dynamics. The first step of the approach is that only a subclass of the shell spacetimes is selected from all spherically symmetric shell spacetimes as described in the previous subsection, by fixing the value, $E$, say, of the Schwarzschild mass $E_{-}$in their internal subspacetimes. Then the spacetime $\mathcal{M}_{1}$ is a fixed, complete Kruskal spacetime and each shell spacetime defines a trajectory $\Sigma_{1}$ in it. This trajectory satisfies Eq. (11) and (3) with $\epsilon=-1$. In Ref. [9] a proof is given that each such trajectory belongs to a unique shell spacetime: the condition that the shell spacetime satisfies the full set of Einstein equations determines the mass $E_{+}$ of the right subspacetime $\mathcal{M}_{+}$and the trajectory $R_{+}(t), T_{+}(t)$ in it up to a constant shift of $T_{+}(t)$. In this sense, the shell dynamics can be reduced to a dynamics of a particle-like object on a fixed two-dimensional spacetime $\mathcal{M}_{1}$. In Ref. [9], the corresponding variational principle was shown to be uniquely determined (up to a coordinate-dependent factor in front of the super-Hamiltonian) if the super-Hamiltonian was required to be at most quadratic in momenta, and the value of the momentum conserved due to the time-shift symmetry to be the negative of the Schwarzschild mass of the external subspacetime $\mathcal{M}_{+}$, that is the total energy of the shell. 
Let us describe this variational principle. We will leave out the indices '-' and ' 1 ' in refering to $\mathcal{M}_{-}$and $\mathcal{M}_{1}$ within the Warsaw approach. The coordinates $x^{a}, a=0,1$ on $\mathcal{M}$ play the role of the canonical coordinates of the system; the corresponding canonical momenta are denoted by $p_{a}, a=0,1$. The action reads

$$
S_{1}=\int d t\left(p_{a} \dot{x}^{a}-\mathcal{N} \mathcal{C}_{1}\right)
$$

where $t$ is an arbitrary parameter along dynamical trajectories, $\mathcal{N}$ is a Lagrange multiplier and the super-Hamiltonian $\mathcal{C}_{1}$ is given by

$$
\mathcal{C}_{1}:=\frac{1}{2} F\left(g^{a b} p_{a} p_{b}+M^{2}\right)-W g^{a b} p_{a} \xi_{b}-\frac{1}{2} W^{2} .
$$

Here, $g^{a b}(x)$ is the contravariant metric on $\mathcal{M}$ (observe that the supermetric $F g^{a b}$ is flat), $\xi^{a}(x)$ is the Schwarzschild time-shift Killing vector on $\mathcal{M}, F=-g^{a b} \xi_{a} \xi_{b}$, the potential $W(x)$ is defined by

$$
W(R):=E-\frac{M^{2}(R)}{2 R}
$$

and $M(R)$ is the mass function of the shell matter.

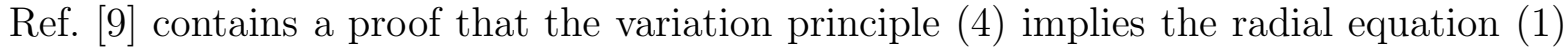
and the time equation (3) with $\epsilon=-1$. The time equation (3) with $\epsilon=+1$ must be added by hand.

We observe that the extended phase space is four-dimensional and that there is one constraint. Hence, the system has just one physical degree of freedom; this can be chosen to be, for example, the radius of the shell.

As one easily verifies,

$$
\left\{\mathcal{C}_{1}, \xi^{a} p_{a}\right\}=0
$$

Thus, $\xi^{a} p_{a}$ is conserved. The value of $\xi^{a} p_{a}$ is $-E_{+}$, where $E_{+}$is the total energy of the shell, or alternatively, the Schwarzschild mass of the external subspacetime of the shell spacetime. Thus, $p_{a}$ cannot be homogeneous in the velocities $\dot{x}^{a}$; this is the reason for the presence of the vector potential $W \xi_{a}$ in the super-Hamiltonian $\mathcal{C}_{1}$. 
To do calculations, we shall choose the Kruskal coordinates $U$ and $V$ for $x^{a}$; let us recapitulate some properties of these coordinates. The spacetime metric has the form

$$
d s^{2}=-\frac{(4 E)^{2}}{\kappa \mathrm{e}^{\kappa}} d U d V
$$

where $\kappa=\kappa(-U V)$, and the function $\kappa(x)$ is defined in the interval $(-1, \infty)$ by its inverse,

$$
\kappa^{-1}(x):=(x-1) \mathrm{e}^{x} ;
$$

this implies the identity

$$
\kappa^{\prime}(x)=\frac{1}{\kappa(x) \mathrm{e}^{\kappa(x)}} .
$$

The transformation to the Schwarzschild coordinates is given by

$$
R=2 E \kappa(-U V), \quad T=2 E \ln \left|\frac{V}{U}\right|
$$

it follows that

$$
F(R)=-\frac{U V}{\kappa \mathrm{e}^{\kappa}}=\frac{\kappa-1}{\kappa} .
$$

The time-shift Killing vector $\xi$ has the form

$$
\xi=\frac{1}{4 E}\left(-U \partial_{U}+V \partial_{V}\right) .
$$

The variational principle (4) and (5) for the Warsaw approach reads in the Kruskal coordinates as follows:

$$
S_{1}=\int d t\left(P_{U} \dot{U}+P_{V} \dot{V}-\mathcal{N C}_{1}\right)
$$

where

$$
\mathcal{C}_{1}=\frac{U V P_{U} P_{V}}{2(2 E)^{2}}+\frac{\kappa-1}{2 \kappa} M^{2}+\frac{W}{4 E}\left(U P_{U}-V P_{V}\right)-\frac{W^{2}}{2} .
$$

Our definitions (5), (10) and (6) of $\mathcal{C}_{1}$ and $W$ differ from those given in Ref. [9] by the factor $F$ so that the super-Hamiltonian $\mathcal{C}_{1}$ and the potential $W$ are smooth everywhere. 
Using Eq. (10) one can show that the function $\mathcal{C}_{1}$ has a non-zero gradient everywhere on the phase space with the exception of the points with $U=V=0$ (whose projections to the configuration space is the crossing point of the two horizons $R=2 E$ ). Moreover, the constraint equation $\mathcal{C}_{1}=0$ has no finite momenta solution there in the generic case $W(2 E) \neq 0$. This is roughly a consequence of the requirement that $\xi^{a} p_{a}=E_{+}$and the fact that all components of the vector $\xi$ vanish at the crossing point. Of course, in the special case of flat spacetime $\left(E_{-}=0\right)$ this problem is avoided. We shall also show in Sec. IIIB that this singularity is due to the choice of the coordinates $x^{a}$ and $p_{a}$.

An analysis that is based on the Kruskal coordinates cannot include the two special cases, $E_{-}=0$ and $E_{+}=0$, in which one of the subspacetimes is flat because the Kruskal coordinates become badly singular in the limit $E_{ \pm} \rightarrow 0$. Especially the case $E_{-}=0$ is, however, important. We give a short description of it in the Appendix.

\section{The Potsdam approach}

In this subsection, another approach to the shell dynamics is described. It starts so to speak from 'the first principles': in Ref. [10], general Lagrangian and Hamiltonian formalisms for massive shell are developed, starting from the Einstein-Hilbert and a fluid actions. The Hamiltonian formalism is reduced in Ref. [11] by spherical symmetry, using a transformation of canonical variables invented by Kuchař [13]. The basic properties of the resulting constrained system are as follows.

The canonical coordinates involved are four coordinates of the shell in the left and the right subspacetimes $\mathcal{M}_{ \pm}$. For example, one can take the Kruskal coordinates $U_{ \pm}$and $V_{ \pm}$. Another variable is the co-called 'Kruskal momentum' $P_{K}^{\epsilon}$ that was introduced in Ref. [11]:

$$
P_{K}^{\epsilon}:=R_{\epsilon} \operatorname{arctanh} \frac{d X_{\epsilon}}{d Z_{\epsilon}}
$$

where $X_{\epsilon}:=-U_{\epsilon}+V_{\epsilon}$ and $Z_{\epsilon}:=U_{\epsilon}+V_{\epsilon}$, so that the argument of the arctanh can be

considered as the 'Kruskal velocity', that is the velocity of the shell with respect to the Kruskal frame $\left(n_{\epsilon}(1,1), n_{\epsilon}(-1,1)\right)\left(n_{\epsilon}\right.$ is a suitable normalization factor). First, 


$$
P_{K}^{\epsilon}=\frac{R_{\epsilon}}{2} \ln \frac{d V_{\epsilon}}{d U_{\epsilon}}
$$

observe that $d V_{\epsilon} / d U_{\epsilon} \geq 0$ holds along each non-spacelike curve. There is a relation of the Kruskal momentum to the proper-time velocity $\left(d U_{\epsilon} / d s, d V_{\epsilon} / d s\right)$; it follows from Eq. (11) and the normalization condition,

$$
-\frac{\left(4 E_{\epsilon}\right)^{2}}{\kappa_{\epsilon} \mathrm{e}^{\kappa_{\epsilon}}} \frac{d U_{\epsilon}}{d s} \frac{d V_{\epsilon}}{d s}=-1
$$

where $\kappa_{\epsilon}:=\kappa\left(-U_{\epsilon} V_{\epsilon}\right)$, namely,

$$
\begin{aligned}
\frac{d U_{\epsilon}}{d s} & =\frac{\sqrt{\kappa_{\epsilon} \mathrm{e}^{\kappa_{\epsilon}}}}{4 E_{\epsilon}} \mathrm{e}^{-P_{K}^{\epsilon} / R_{\epsilon}}, \\
\frac{d V_{\epsilon}}{d s} & =\frac{\sqrt{\kappa_{\epsilon} \mathrm{e}^{\kappa_{\epsilon}}}}{4 E_{\epsilon}} \mathrm{e}^{P_{K}^{\epsilon} / R_{\epsilon}} .
\end{aligned}
$$

The variational principle of the Potsdam approach has been written down in terms of Kruskal coordinates $U_{\epsilon}, V_{\epsilon}$, Kruskal momenta $P_{K}^{\epsilon}$ and the energies $E_{\epsilon}$ in Ref. [11]:

$$
S_{2}=\int d t\left(\left[-2 E \kappa \dot{P}_{K}+E^{2}(\kappa+1) \mathrm{e}^{-\kappa}(V \dot{U}-U \dot{V})\right]+2 \tilde{\nu}[E \kappa]-\nu \mathcal{C}_{2}\right)
$$

where

$$
\mathcal{C}_{2}:=\left[E \sqrt{\kappa} \mathrm{e}^{-\kappa / 2}\left(-U \mathrm{e}^{P_{K} / R}+V \mathrm{e}^{-P_{K} / R}\right)\right]+M\left(E_{+} \kappa_{+}+E_{-} \kappa_{-}\right)
$$

the symbol $[f]$ denotes the jump of the quantity $f$ across the shell, $[f]:=f_{+}-f_{-}$and $\bar{f}$ denotes the average of the values of $f$ from left and right: $\bar{f}:=\left(f_{+}+f_{-}\right) / 2$.

Let us stress that the origin of the symplectic structure defined by the Liouville form in action (15) lies in the form of the Einstein-Hilbert Lagrangian. As concerns the constraints, there are three of them in all: the two primaries, $R_{+}-R_{-}=0$ and $\mathcal{C}_{2}=0$, and one secondary, $\chi=0$, which can be obtained by variation with respect to $P_{K \pm}$ after employing the constraint $E_{+} \kappa_{+}=E_{-} \kappa_{-}$:

$$
\chi:=\frac{\partial C_{2}}{\partial P_{K}^{+}}+\frac{\partial C_{2}}{\partial P_{K}^{-}}
$$

We obtain 


$$
\chi=-\left[\frac{1}{2 \sqrt{\kappa \mathrm{e}^{\kappa}}}\left(U \mathrm{e}^{P_{K} / R}+V \mathrm{e}^{-P_{K} / R}\right)\right] .
$$

The pair $\left(\chi, R_{+}-R_{-}\right)$form the second-class part of the constraints set (see Ref. [11]).

Hence, we have an eight-dimensional extended phase space and three constraints. This implies that there are two physical degrees of freedom; they can e.g. be chosen as $E_{-}$and $R_{-}$. This should be compared with the Warsaw approach of the previous subsection, where the Schwarzschild mass $E_{-}$was just a parameter having vanishing Poisson brackets with everything.

The Kruskal metric is invariant with respect to the one-dimensional transformation group $g_{\lambda}, \lambda \in(-\infty, \infty):$

$$
U \mapsto U \mathrm{e}^{\lambda}, \quad V \mapsto V \mathrm{e}^{-\lambda}
$$

From this isometry, an infinite-dimensional abelian group of symmetry for the action (15) can be constructed as follows. First, it can transform the coordinates $U_{-}, V_{-}$of the left and $U_{+}, V_{+}$of the right subspacetimes independently. Second, this double transformation can be performed for each value of the pair $\left(E_{+}, E_{-}\right)$independently. Such a transformation has the form

$$
U_{\epsilon} \mapsto U_{\epsilon} \mathrm{e}^{\lambda_{\epsilon}\left(E_{\epsilon}\right)}, \quad V_{\epsilon} \mapsto V_{\epsilon} \mathrm{e}^{-\lambda_{\epsilon}\left(E_{\epsilon}\right)}, \quad \epsilon= \pm 1
$$

where $\lambda_{-}\left(E_{-}\right)$and $\lambda_{+}\left(E_{+}\right)$are two arbitrary (smooth) functions. Let us denote the transformation by $G\left[\lambda_{-}\left(E_{-}\right), \lambda_{+}\left(E_{+}\right)\right]$. Clearly, $G$ preserves the products $U_{\epsilon} V_{\epsilon}$ and the two masses $E_{\epsilon}$ 列.

\footnotetext{
${ }^{3}$ The origin of this symmetry is easy to understand: continuous symmetry groups are generated by perennials (constants of motion) ; $E_{-}$and $E_{+}$are perennials, and any functions $\Lambda\left(E_{-}\right)$and $\Lambda\left(E_{+}\right)$are also perennials. Thus, one obtains a symmetry depending on two arbitrary functions of one variable. In fact, the symmetry group is even larger: it is generated by all perennials of the form $\Lambda\left(E_{+}, E_{-}\right)$!
} 
The corresponding transformation of the Kruskal momenta is determined by Eq. (11):

$$
P_{K}^{\epsilon} \mapsto P_{K}^{\epsilon}-R \lambda_{\epsilon}\left(E_{\epsilon}\right)
$$

Then, the functions $U_{\epsilon} \mathrm{e}^{P_{K}^{\epsilon} / R_{\epsilon}}$ and $V_{\epsilon} \mathrm{e}^{-P_{K}^{\epsilon} / R_{\epsilon}}$ are invariants, and so are both constraints, $\mathcal{C}_{2}$ and $R_{+}-R_{-}$. A little more difficult is to see that the Liouville form of the action (15) changes at most by a closed form. Let us write out the transformation of just the $\epsilon$-part of it leaving out the index $\epsilon$

$$
\begin{aligned}
& -R d P_{K}+E^{2}(1+\kappa) \mathrm{e}^{-\kappa}(V d U-U d V) \rightarrow \\
& -R d P_{K}+E^{2}(1+\kappa) \mathrm{e}^{-\kappa}(V d U-U d V)+R d(R \lambda)+2 E^{2}(1+\kappa) \mathrm{e}^{-\kappa} U V d \lambda .
\end{aligned}
$$

Using Eq. (8) and $R=2 E \kappa$, we obtain for the last two terms:

$$
R d(R \lambda)+2 E^{2}(1+\kappa) \mathrm{e}^{-\kappa} U V d \lambda=R \lambda d R+\frac{R^{2}}{2} d \lambda+2 E^{2} \frac{d \lambda}{d E} d E
$$

which is a closed form.

The Kruskal spacetime also admits an isometry group of four elements with generators $T_{1}$ and $T_{2}$, the two transformations that are obtained by extending the Schwarzschild time reversal $T \mapsto-T$, either from the quadrant $U<0, V>0$ or from $U>0, V>0$ to the whole of the Kruskal manifold:

$$
T_{1}(U, V)=(-V,-U), \quad T_{2}(U, V)=(V, U) .
$$

These transformations can be used to define symmetries of the variational principle (15) as follows. Consider first $T_{1}$; it changes the time orientation of the Kruskal spacetime. Thus, it must act in both Kruskal subspacetimes of each shell spacetime simultaneously, or else the resulting shell spacetime has no time orientation. We have, therefore

$$
T_{1}\left(U_{\epsilon}, V_{\epsilon}\right)=\left(-V_{\epsilon},-U_{\epsilon}\right)
$$

The action of this transformation on the momentum $P_{K}^{\epsilon}$ is determined by Eq. (11):

$$
P_{K}^{\epsilon} \mapsto-P_{K}^{\epsilon}
$$


Finally, $E_{\epsilon}$ are clearly invariants. This defines a transformation in the extended phase space that will be also denoted by $T_{1}$. Observe that the constraints $\mathcal{C}_{2}$ and $R_{+}-R_{-}$are invariant, but that the Liouvile form in the action (15) changes sign. Thus, $T_{1}$ is an anti-symplectic map (this is common for time reversals).

Next, consider $T_{2}$; it changes the space orientation exchanging right and left. Thus, we have to define:

$$
T_{2}\left(U_{\epsilon}, V_{\epsilon}\right)=\left(V_{-\epsilon}, U_{-\epsilon}\right),
$$

and, similarly,

$$
P_{K}^{-} \leftrightarrow-P_{K}^{+}, \quad E_{-} \leftrightarrow E_{+} .
$$

One can see immediately that both the constraints and the Liouville form are invariant with respect to this transfromation.

\section{CARTAN FORMS}

\section{A. Reparametrization-invariant reduction method}

The two variational principles (91) and (15) seem to be as different as one can imagine. Although they depend on some common variables (because $U=U_{-}$and $V=V_{-}$), the momenta are different in each case, and the nature of the super-Hamiltonians (10) and (16) is very different: $\mathcal{C}_{1}=0$ is a condition on the norm of the two-velocity of the shell in the left subspacetime, whereas $\mathcal{C}_{2}=0$ relates the time-time-components of the second fundamental forms of the shell in the left and right subspacetime (indeed, $\mathcal{C}_{2}=0$ is a component of the Israel equation).

It turns out that the simplest way to compare the two systems is to solve all constraints thus reducing the systems completely. It is, however, the kind of reduction that does not

deparametrize the system: no choice of gauge is necessary. The resulting Lagrangian is the 
value $\Theta(\dot{X})$ of the so-called Cartan form $\Theta$ at the tangent vector $\dot{X}$ of a trajectory, and so it is homogeneous in velocities. The variation of the corresponding action,

$$
S=\int d t \Theta(\dot{X})
$$

leads to dynamical equations of the form

$$
d \Theta(\dot{X}, \delta X)=0, \quad \forall \delta X
$$

$d \Theta$ coincides with the presymplectic form $\Omega(\cdot, \cdot)$ on the constraint surface of the system, and the equation of motion (20) simply says that $\dot{X}$ must lie in the degeneracy subspace of $\Omega$ (for more about presymplectic forms see, e.g. Refs. [14] and [15], cf. also [16]).

\section{B. Warsaw description}

In this subsection, we will reduce the Warsaw system. Since the Potsdam approach was written down only for massive shells as yet, we restrict ourselves to massive shells, $M(R) \neq 0$,

in the rest of the paper. We observe then that the constraint $\mathcal{C}_{1}=0$ can be easily solved, if we express the momenta $P_{U}$ and $P_{V}$ by means of $P_{K}$ as follows. The variation of the action (9) with respect to the momenta gives their relation to the velocities:

$$
\begin{aligned}
& P_{U}=\frac{2(2 E)^{2}}{U V}\left(\frac{\dot{U}}{\mathcal{N}}-\frac{W U}{4 E}\right), \\
& P_{V}=\frac{2(2 E)^{2}}{U V}\left(\frac{\dot{V}}{\mathcal{N}}+\frac{W V}{4 E}\right) .
\end{aligned}
$$

If we insert these expressions for the momenta into the constraint (10), we obtain that

$$
\frac{\dot{U}}{\mathcal{N}} \cdot \frac{\dot{V}}{\mathcal{N}}=\frac{(\kappa-1)^{2} \mathrm{e}^{\kappa}}{\kappa} \cdot \frac{M^{2}}{(4 E)^{2}}
$$

A comparison with Eq. (12) and a use of Eqs. (13) and (14) leads to

$$
\begin{aligned}
& P_{U}=2 E\left(-\frac{M}{\sqrt{\kappa \mathrm{e}^{\kappa}}} \mathrm{e}^{P_{K} / R}+\frac{W}{U}\right), \\
& P_{V}=2 E\left(-\frac{M}{\sqrt{\kappa \mathrm{e}^{\kappa}}} \mathrm{e}^{-P_{K} / R}-\frac{W}{V}\right) .
\end{aligned}
$$


One easily verifies that these expressions for $P_{U}$ and $P_{V}$ satisfy the constraint (10) identically. In this way, we have arrived at the constraint manifold $\Gamma_{1}$ with the coordinates $U, V$ and $P_{K}$. On this manifold, there are two important quantities: the pull-back $-E_{+}$of the conserved quantity $\xi^{a} p_{a}=1 /(4 E)\left(-U P_{U}+V P_{V}\right)$ and the Cartan form. Substituting for the momenta $P_{U}$ and $P_{V}$, we obtain:

$$
E_{+}=E+\frac{M(R)}{2 \sqrt{\kappa \mathrm{e}^{\kappa}}}\left(-U \mathrm{e}^{P_{K} / R}+V \mathrm{e}^{-P_{K} / R}\right)-\frac{M^{2}(R)}{2 R},
$$

where $R$ is an abbreviation for $2 E \kappa(-U V)$. The Cartan form is obtained, if we substitute for the momenta into the Liouville form of the action (9):

$$
\Theta_{1}=2 E W d\left(\ln \left|\frac{U}{V}\right|\right)-\frac{2 E M(R)}{\sqrt{\kappa \mathrm{e}^{\kappa}}}\left(\mathrm{e}^{P_{K} / R} d U+\mathrm{e}^{-P_{K} / R} d V\right) .
$$

One would expect that these objects would be singular at the point $U=V=0$ where the horizons cross, and indeed, the Cartan form (24) diverges even at both horizons $U=0$ and $V=0$. However, this singularity can be removed by subtracting the differential of the function

$$
2 E W(2 E) \ln \left|\frac{U}{V}\right|
$$

in this way, we obtain an everywhere regular Cartan form

$$
\Theta_{1 r}=2 E W_{r}(V d U-U d V)-\frac{2 E M}{\sqrt{\kappa \mathrm{e}^{\kappa}}}\left(\mathrm{e}^{P_{K} / R} d U+\mathrm{e}^{-P_{K} / R} d V\right)
$$

where

$$
W_{r}(-U V):=\frac{W(R)-W(2 E)}{U V}
$$

is a smooth function. For example, with $M=$ const,

$$
W_{r}=\frac{M^{2}}{4 E \kappa \mathrm{e}^{\kappa}} .
$$

The regularity of the constraint system requires, however, a slightly stronger condition that just the smoothness of the Cartan form: the presymplectic form $d \Theta_{1}=d \Theta_{1 r}$ must 
be smooth and its degeneracy subspace must be one-dimensional everywhere in $\Gamma_{1}$. Let us calculate the presymplectic form; a straightforward but tedious procedure yields:

$$
\begin{aligned}
d \Theta_{1} & =\left(\frac{2(2 E)^{2} W_{R}}{\kappa \mathrm{e}^{\kappa}}+\frac{2 E}{\kappa \mathrm{e}^{\kappa}}\left(\frac{M}{\sqrt{\kappa \mathrm{e}^{\kappa}}}\right)_{\kappa}\left(-U \mathrm{e}^{P_{K} / R}+V \mathrm{e}^{-P_{K} / R}\right)\right. \\
& \left.+\frac{M P_{K}}{\kappa^{3} \mathrm{e}^{\kappa} \sqrt{\kappa \mathrm{e}^{\kappa}}}\left(U \mathrm{e}^{P_{K} / R}+V \mathrm{e}^{-P_{K} / R}\right)\right) d U \wedge d V \\
& -\frac{M}{\kappa \sqrt{\kappa \mathrm{e}^{\kappa}}}\left(\mathrm{e}^{P_{K} / R} d P_{K} \wedge d U-\mathrm{e}^{-P_{K} / R} d P_{K} \wedge d V\right),
\end{aligned}
$$

where the indices $R$ and $\kappa$ denote partial derivatives with respect to the corresponding variables. An inspection shows that this form is smooth and non-zero everywhere; this is sufficient for a two form on a three-dimensional manifold to define a smooth one-dimensional degeneracy distribution. Thus, the constraint system $\left(\Gamma_{1}, \Theta_{1}\right)$ is completely regular even at $U=V=0$. The singularity of the variational principle (4) is due just to the choice of the variables $x^{a}$ and $p_{a}$ : the simplicity of the super-Hamiltonian (5) is traded for the singularity at $U=V=0$. In any case, the constraint manifold $\Gamma_{1}$ is defined by the coordinates $U, V$, and $P_{K}$ in the ranges

$$
-U V \in(-1, \infty), \quad E_{+} \in(0, \infty)
$$

\section{Symmetry and adapted coordinates}

In this section, we shall study the static symmetry of the Kruskal spacetime and the corresponding symmetry of the system $\left(\Gamma_{1}, \Theta_{1}\right)$. We shall use the symmetry to find some coordinate systems which will simplify the subsequent calculations.

Eqs. (17), and (19) for $\lambda=$ const, define a transformation on $\Gamma_{1}$; this transformation leaves the function $E_{+}$and the form $\Theta_{1}$ invariant. Thus, it is a symmetry of the constraint

system. From the discrete transformations, only $T_{1}$ survives; $T_{2}$ would map our system to an equivalent one, which would be obtained if we based the description on the trajectory in the right subspacetime $\mathcal{M}_{2}$. It is quite natural that this much smaller transformation 
group remained from the group $G\left[\lambda_{+}\left(E_{+}\right), \lambda_{-}\left(E_{-}\right)\right]$here, because the right subspacetime is missing and $E=$ const, so $\lambda(E)=$ const. We call this transformation $G(\lambda)$.

The orbits of the group $G(\lambda)$ in $\Gamma_{1}$ are one-dimensional, and the quotient $\Gamma_{1} / G(\lambda)$ is a two-dimensional manifold. It is useful to introduce coordinate systems that are adapted to this quotient structure: two coordinates that are constant along the group orbits, and another one that transforms simply by the group.

\section{Coordinates $u, v$ and $\tilde{P}$}

Let us define:

$$
u:=\frac{U \mathrm{e}^{P_{K} / R}}{\sqrt{\kappa \mathrm{e}^{\kappa}}}, \quad v:=\frac{V \mathrm{e}^{-P_{K} / R}}{\sqrt{\kappa \mathrm{e}^{\kappa}}} .
$$

The factor $1 / \sqrt{\kappa \mathrm{e}^{\kappa}}$ does not improve the transformation properties, but it leads to additional simplifications: indeed, all exponentials, square roots and transcendental Kruskal functions will disappear. Futher,

$$
\tilde{P}:=\frac{E P_{K}}{R},
$$

hence

$$
\tilde{P} \mapsto \tilde{P}-E \lambda
$$

Eq. (8) implies that

$$
u v=-1+\frac{2 E}{R}
$$

or

$$
R=\frac{2 E}{1+u v}
$$

and this, together with Eq. (7) yield

$$
\kappa=\frac{1}{1+u v}
$$


The objects $E_{+}$and $\Theta_{1}$ can be transformed into these coordinates with the results:

$$
E_{+}=E-\frac{M(R)}{2}(u-v)-\frac{M^{2}(R)}{2 R},
$$

and

$$
\begin{gathered}
\Theta_{1}=-\frac{E M^{2}(R)}{R} d\left(\ln \left|\frac{u}{v}\right|\right)-2 E M(R) d(u+v) \\
-\frac{M(R)}{2}(u+v)\left(1+\frac{2 E}{R}\right) d R-4 E_{+} d \tilde{P}
\end{gathered}
$$

where $R$ is defined by Eq. (28).

\section{Coordinates $R, E_{+}$and $\tilde{P}$}

The function $E_{+}$is not only invariant with respect to the group $G(\lambda)$, but also an integral of motion. Hence, projections of the dynamical trajectories to the quotient $\Gamma_{1} / G(\lambda)$ are just the curves $E_{+}=$const. It will prove advantageous, therefore, to transform to the coordinates $R$ and $E_{+}$on the quotient. Of course, $R$ and $E_{+}$are not everywhere regular coordinates: clearly, at the maximal radii of bound trajectories, gradients of both functions $R$ and $E_{+}$ vanish in the direction of the trajectories; we can, however, still work with these coordinates in the rest of the quotient, and match the results at the singular points. The transformation from $u$ and $v$ to $E_{+}$and $R$ are given by Eqs. (30) and (28). The inverse transformation is obtained by solving these equations for $u$ and $v$ :

$$
\begin{aligned}
& u_{\omega}=\frac{-Y+\omega \sqrt{Y^{2}-4 X}}{2}, \\
& v_{\omega}=\frac{Y+\omega \sqrt{Y^{2}-4 X}}{2},
\end{aligned}
$$

where $\omega$ is a sign, whose significance will be established shortly,

$$
\begin{aligned}
& X:=1-\frac{2 E}{R}, \\
& Y:=2\left(\frac{E_{+}-E}{M(R)}+\frac{M(R)}{2 R}\right),
\end{aligned}
$$

and 


$$
Y^{2}-4 X=-4 V(R)
$$

where $V(R)$ is given by Eq. (2). Thus, if $V(R)$ vanishes, $\dot{R}$ must also vanish and this happens only at the turning point of a bound trajectory. The meaning of the sign $\omega$ in front of the square root is simple. As

$$
\begin{aligned}
(-u+v)_{\omega} & =Y, \\
(u+v)_{\omega} & =\omega \sqrt{Y^{2}-4 X},
\end{aligned}
$$

it distinguishes the upper and lower half of the $u$-v-plane; the turning points are lying at $u+v=0$ and the coordinates $E_{+}$and $R$ are not regular there.

\section{Potsdam description}

In this subsection, we reduce our second system completely. In analogy with Eq. (25), we define

$$
u_{\epsilon}:=\frac{U_{\epsilon} \mathrm{e}^{P_{K}^{\epsilon} / R_{\epsilon}}}{\sqrt{\kappa_{\epsilon} \mathrm{e}^{\kappa_{\epsilon}}}}, \quad v_{\epsilon}:=\frac{V_{\epsilon} \mathrm{e}^{-P_{K}^{\epsilon} / R_{\epsilon}}}{\sqrt{\kappa_{\epsilon} \mathrm{e}^{\kappa_{\epsilon}}}}
$$

and obtain the relations

$$
u_{\epsilon} v_{\epsilon}=-1+\frac{2 E_{\epsilon}}{R_{\epsilon}}, \quad R_{\epsilon}=\frac{2 E_{\epsilon}}{1+u_{\epsilon} v_{\epsilon}}, \quad \kappa_{\epsilon}=\frac{1}{1+u_{\epsilon} v_{\epsilon}}
$$

The three constraints $\mathcal{C}_{2}=0, R_{+}=R_{-}$and $\chi=0$ simplify greatly in these coordinates:

$$
\begin{aligned}
\mathcal{C}_{2} & =\frac{1}{2}[R(-u+v)]+M(\bar{R}), \\
\chi & =\frac{1}{2}[u+v], \\
R & =R_{+}=R_{-} .
\end{aligned}
$$

They can be solved immediately, either for $u_{+}, v_{+}$and $E_{+}$:

$$
\begin{aligned}
& u_{+}=u_{-}+\frac{M(R)}{R}, \\
& v_{+}=v_{-}-\frac{M(R)}{R}, \\
& E_{+}=E_{-}-\frac{M(R)}{2}\left(u_{-}-v_{-}\right)-\frac{M^{2}(R)}{2 R},
\end{aligned}
$$


or for $u_{-}, v_{-}$and $E_{-}$:

$$
E_{-}=E_{+}+\frac{M(R)}{2}\left(u_{+}-v_{+}\right)-\frac{M^{2}(R)}{2 R}
$$

(solutions for $u_{-}$and $v_{-}$are given by Eqs. (37) and (38)).

From the definitions Eqs. (35), we obtain

$$
\ln \left|\frac{U_{\epsilon}}{V_{\epsilon}}\right|=-\frac{2 P_{K}^{\epsilon}}{R}+\ln \left|\frac{u_{\epsilon}}{v_{\epsilon}}\right| .
$$

To calculate the Cartan form $\Theta_{2}$, we first express the Liouville form of the action (15) in the variables $u_{-}, v_{-}, P_{K}^{-}, E_{-}, u_{+}, v_{+}, P_{K}^{+}$and $E_{+}$using an analogy of Eq. (8)

$$
u_{\epsilon} v_{\epsilon}=\left(1-\kappa_{\epsilon}\right) \mathrm{e}^{\kappa_{\epsilon}}
$$

after substituting for $E_{+}, u_{+}$and $v_{+}$and employing several times the equations $R=$ $2 E_{-} \kappa_{-}=2 E_{+} \kappa_{+}$we obtain:

$$
\Theta_{2} \equiv-\left[\left(\frac{R^{2}}{4}-E^{2}\right) d\left(\ln \left|\frac{u}{v}\right|\right)+4 E d \tilde{P}\right]
$$

where the sign ' $\equiv$ ' suggests that we have added some closed forms, and

$$
\tilde{P}_{\epsilon}:=\frac{E_{\epsilon} P_{K}^{\epsilon}}{R}
$$

The form is well-defined and smooth everywhere on the constraint manifold $\Gamma_{2}$ that is defined by the ranges of the coordinates $u_{-}, v_{-}, E_{-}, \tilde{P}_{-}$and $\tilde{P}_{+}$:

$$
\begin{gathered}
u_{-} v_{-} \in(-1, \infty), \quad u_{+} v_{+} \in(-1, \infty), \quad E_{-} \in(0, \infty), \\
E_{+} \in(0, \infty), \quad \tilde{P}_{-} \in(-\infty, \infty), \quad \tilde{P}_{+} \in(-\infty, \infty) .
\end{gathered}
$$

The reduced action has the form

$$
S_{2 C}=\int d t L_{2 C}
$$

where the Lagrangian is 


$$
L_{2 C}=-\left[\left(\frac{R^{2}}{4}-E^{2}\right)\left(\frac{\dot{u}}{u}-\frac{\dot{v}}{v}\right)+4 E \dot{\tilde{P}}\right] .
$$

The symmetry group of the system $\left(\Gamma_{2}, \Theta_{2}\right)$ contains the whole infinite-dimensional group $G\left[\lambda_{-}\left(E_{-}\right), \lambda_{+}\left(E_{+}\right)\right]$. In the coordinates $E_{-}, u_{-}, v_{-}, \tilde{P}_{-}$and $\tilde{P}_{+}$, this transformations take on the from:

$$
E_{-} \mapsto E_{-}, \quad u_{-} \mapsto u_{-}, \quad v_{-} \mapsto v_{-}, \quad \tilde{P}_{-} \mapsto \tilde{P}_{-}-E_{-} \lambda\left(E_{-}\right), \quad \tilde{P}_{+} \mapsto \tilde{P}_{+}-E_{+} \lambda\left(E_{+}\right)
$$

$\Theta_{2}$ changes by $4 E_{+} d\left(E_{+} \lambda_{+}\left(E_{+}\right)\right)-4 E_{-} d\left(E_{-} \lambda\left(E_{-}\right)\right)$, which is obviously a closed form. From the discrete group, $T_{1}$ remains simple; it acts as follows:

$$
E_{-} \mapsto E_{-}, \quad u_{-} \mapsto-u_{-}, \quad v_{-} \mapsto-v_{-}, \quad \tilde{P}_{-} \mapsto-\tilde{P}_{-}, \quad \tilde{P}_{+} \mapsto-\tilde{P}_{+}
$$

\section{EXCLUSION OF A CYCLIC COORDINATE}

If one compares the systems $\left(\Gamma_{1}, \Theta_{1}\right)$ and $\left(\Gamma_{2}, \Theta_{2}\right)$ the first difference that catches one's eye is that $\Gamma_{2}$ is five- and $\Gamma_{1}$ only three-dimensional. In this section, we shall get rid of two dimensions of $\Gamma_{2}$. The fact that the variable $E$ appears in $\left(\Gamma_{1}, \Theta_{1}\right)$ as a parameter implies that we have, as the first step, to select the submanifold $\Gamma_{2 E} \subset \Gamma_{2}$ of constant coordinate $E_{-}$ in $\Gamma_{2}$. As $E_{-}$is a constant of motion, the dynamical trajectories that intersect $\Gamma_{2 E}$ all remain in $\Gamma_{2 E}$. However, the dimension of $\Gamma_{2 E}$ is still larger by 1 than that of $\Gamma_{2}$; moreover, the pull-back $\Theta_{2 E}$ of $\Theta_{2}$ to $\Gamma_{2 E}$ defines a presymplectic form $d \Theta_{2 E}$ that has a two-dimensional space of degeneracy: it contains the vector $\partial / \partial \tilde{P}_{-}$in addition to the direction of motion. Thus, in the second step, we have to construct the manifold $\Gamma_{E}:=\Gamma_{2 E} / \tilde{P}_{-} ;$it is the quotient of $\Gamma_{2 E}$ by the $\tilde{P}_{-}$-curves (i.e. curves with $u_{-}=$const, $v_{-}=$const and $\tilde{P}_{+}=$const). A form $\Theta_{2 E}^{\prime}$ that differs from $\Theta_{2 E}$ by a form closed on $\Gamma_{2 E}$ does not contain the variable $\tilde{P}_{-}$; it can, therefore, be pushed forward by the quotient projection $\pi$ to $\Gamma_{E}$ giving a form that we call $\Theta_{E} \cdot d \Theta_{E}$ is a presymplectic form on $\Gamma_{E}$ with a one-dimensional degeneracy subspace everywhere, and the integral manifolds of this subspaces coincide with the projection to $\Gamma_{E}$ of the original dynamical trajectories. Observe that we have to take a quotient so that 
$\tilde{P}_{-}$remains arbitrary; we are not allowed to choose a surface transversal to the $\tilde{P}_{-}$-curves instead, defined for example by the equation $\tilde{P}_{-}=$const, for $\tilde{P}_{-}$is not a gauge coordinate or an integral of motion (it satisfies a non-trivial dynamical equation that would be violated by $\tilde{P}_{-}=$const $)$.

In terms of coordinates and dynamical equations, the construction is very simple. The pull-back $\Theta_{2 E}$ of $\Theta_{2}$ to $\Gamma_{2 E}$ coincides with $\Theta_{2}$ in the coordinates $u_{-}, v_{-}, \tilde{P}_{-}$and $\tilde{P}_{+}$; only $E_{-}$changes its status: it becomes a constant parameter. Then the term $4 E_{-} d \tilde{P}_{-}$is the differential of the function $4 E_{-} \tilde{P}_{-}$on $\Gamma_{2 E}$ and can be omitted because it does not contribute to $d \Theta_{2 E}$; thus, we end up with the form

$$
\Theta_{2 E}^{\prime}=-\left[\left(\frac{R^{2}}{4}-E^{2}\right) d\left(\ln \left|\frac{u}{v}\right|\right)\right]-4 E_{+} d \tilde{P}_{+}
$$

this form is independent of $\tilde{P}_{-}$and so can be pushed forward to $\Gamma_{E}$; in the coordinates $u_{-}$, $v_{-}$and $\tilde{P}_{+}$, the push-forward is given by the same expression as $\Theta_{2 E}$. Thus, the new action reads

$$
S_{E}=\int d t L_{E},
$$

where the Lagrangian is

$$
L_{E}=-\left[\left(\frac{R^{2}}{4}-E^{2}\right)\left(\frac{\dot{u}}{u}-\frac{\dot{v}}{v}\right)\right]-4 E_{+} \dot{\tilde{P}}_{+}
$$

on the space $\Gamma_{E}$ with coordinates $u_{-}, v_{-}$and $\tilde{P}_{+}$. Let us denote this three-dimensional system by $\left(\Gamma_{E}, \Theta_{E}\right)$. The symmetry of this system is still infinitely-dimensional: it is the transformation group $G\left[\lambda_{+}\left(E_{+}\right), \lambda\right.$ ) (the $\epsilon=+1$-part of the transformation acts only on $\tilde{P}_{+}$ and $\lambda_{-}\left(E_{-}\right)$is a constant, because $E_{-}$is) and the time reversal $T_{1}$.

Let us compare the dynamical equations obtained by varying the actions $S_{2 C}$ and $S_{E}$. The following observation is helpful: the part of the Lagrangian (44) that contains the variables $u_{-}, v_{-}$and $\tilde{P}_{+}$coincides with the Lagrangian (46). It follows that the variations of action $S_{2 C}$ with respect to the variables $u_{-}, v_{-}$and $\tilde{P}_{+}$differ from the corresponding variations of the action $S_{E}$ only by terms that are proportional to $\dot{E}_{-}$; but $\dot{E}_{-}=0$ is the 
dynamical equation obtained by varying the action $S_{2 C}$ with respect to $\tilde{P}_{-}$. Hence, the three dynamical equations of the system $\left(\Gamma_{2}, \Theta_{2}\right)$ due to variation of $u_{-}, v_{-}$and $\tilde{P}_{-}$, if one sets $\dot{E}_{-}=0$ in them, are identical with the corresponding three equations of the system $\left(\Gamma_{E}, \Theta_{E}\right)$. The equation $\dot{E}_{-}=0$ is free in the system $\left(\Gamma_{E}, \Theta_{E}\right)$, because $E_{-}$is a constant parameter there. Finally, there is another equation in the system $\left(\Gamma_{2}, \Theta_{2}\right)$, namely that due to variation of $E_{-}$; it can be written in the form

$$
4 \dot{\tilde{P}}_{-}=-\frac{\partial L_{E}}{\partial E_{-}}
$$

it cannot be obtained in the system $\left(\Gamma_{E}, \Theta_{E}\right)$, because $\tilde{P}_{-}$is the cyclic coordinate that has been eliminated, and the new variation principle does not contain any information about it. We observe that $\tilde{P}_{-}$is 'completely smeared' even classicaly, as any point of $\Gamma_{E}$ is a whole $\tilde{P}_{-}$-curve, $\tilde{P}_{-} \in(-\infty,+\infty)$, and the remaining variables in the system $\left(\Gamma_{E}, \Theta_{E}\right)$, namely $u_{-}, v_{-}$and $\tilde{P}_{+}$have vanishing Poisson brackets with $E_{-}$in the original Potsdam system.

This is in a nice correspondence with the quantum mechanics construted for the two classical systems (if the factor-ordering problem is solved suitably): the Hilbert space $\mathcal{H}$ of the system $\left(\Gamma_{2}, \Theta_{2}\right)$ can be written as the orthogonal sum of the eigenspaces $\mathcal{H}_{E_{-}}$of the operator $\hat{E}_{-} ; E_{-}$is a point of its spectrum $\sigma\left(\hat{E}_{-}\right)$. On the Hilbert space $\mathcal{H}_{E_{-}}$, only those elements of the algebra of observables can act that commute with $\hat{E}_{-}$, and $\mathcal{H}_{E_{-}}$is the Hilbert space of the system $\left(\Gamma_{E}, \Theta_{E}\right)$.

We can even reconstruct a trajectory of the system $\left(\Gamma_{2}, \Theta_{2}\right)$ from one of $\left(\Gamma_{E}, \Theta_{E}\right)$ using the equation (47) which must simply be added by hand, as follows. Let $u_{-}(t), v_{-}(t)$ and $\tilde{P}_{+}(t)$ be a trajectory of $\left(\Gamma_{E}, \Theta_{E}\right)$ parametrized by an arbitrary parameter $t$. Let us substitute these functions for $u_{-}, v_{-}$and $\tilde{P}_{+}$in Eq. (47). We obtain an equation of the form

$$
\frac{d \tilde{P}_{-}}{d t}=-\frac{1}{4} \frac{\partial L_{E}}{\partial E_{-}}\left(u_{-}(t), v_{-}(t), \tilde{P}_{+}(t)\right)
$$

the integration will yield the function $\tilde{P}_{-}(t)$ depending on one arbitrary constant (this constant is determined by an initial value of $\left.\tilde{P}_{-}\right)$. Such a procedure is, in fact, equivalent to reconstructing the manifold $\Gamma_{2}$ from $\Gamma_{E}$ by 


$$
\Gamma_{2}=\Gamma_{E} \times(-\infty,+\infty)_{E_{-}} \times(-\infty,+\infty)_{\tilde{P}_{-}}
$$

and adding the term $4 E_{-} d \tilde{P}_{-}$to $\Theta_{E}$. In the quantum mechanics, this corresponds to defining

$$
\mathcal{H}:=\sum_{\sigma\left(\hat{E}_{-}\right)} \otimes_{\perp} \mathcal{H}_{E_{-}}
$$

(in general, one has to be careful about the spectrum of the conserved quantity; this is here just $(-\infty,+\infty))$ and

$$
\hat{\tilde{P}}_{-}:=\frac{i}{4} \frac{\partial}{\partial E_{-}}
$$

the $E_{-}$-dependence of the Hamiltonian of the system $\left(\Gamma_{E}, \Theta_{E}\right)$ will then lead to some time evolution of $\hat{\tilde{P}}_{-}$.

\section{SEARCH FOR THE EQUIVALENCE MAP}

It remains to find a transformation between the system $\left(\Gamma_{E}, \Theta_{E}\right)$ and $\left(\Gamma_{1}, \Theta_{1}\right)$ showing that they are equivalent. This problem will be studied in the present section.

The basic properties of such a map (which is, in fact a 'morphism of presymplectic manifolds'), let us denote it by $\Phi$, are the following

1. $\Phi: \Gamma_{1} \mapsto \Gamma_{E}$ is a diffeomorphism.

2. The pull-back $\Phi_{*} \Theta_{E}$ of $\Theta_{E}$ to $\Gamma_{1}$ differs from $\Theta_{1}$ by a closed form. This guarantees that the presymplectic forms coincidet.

3. If $f_{1}: \Gamma_{1} \mapsto \mathbf{R}$ and $f_{E}: \Gamma_{E} \mapsto \mathbf{R}$ are quantities with the same physical or geometrical significance in both systems, then $\Phi_{*} f_{E}=f_{1}$.

\footnotetext{
${ }^{4}$ To define equivalent dynamics, two presymplectic forms just had to be proportional to each other with an arbitrary function on the constraint surface as the factor of proportionality. However, presymplectic forms contain more information: they define the Poisson brackets of perennials (that is, functions that are constant along the trajectories, cf. [16]); thus, the factor must be equal to 1 .
} 
Suppose that such a map exists. Let us express it by means of the coordinates $(u, v, \tilde{P})$ on $\left(\Gamma_{1}, \Theta_{1}\right)$ and $\left(u_{-}, v_{-}, \tilde{P}_{+}\right)$on $\left(\Gamma_{E}, \Theta_{E}\right)$ :

$$
\begin{aligned}
& u_{-}=u_{-} \circ \Phi(u, v, \tilde{P}), \\
& v_{-}=v_{-} \circ \Phi(u, v, \tilde{P}), \\
& \tilde{P}_{+}=\tilde{P}_{+} \circ \Phi(u, v, \tilde{P}) .
\end{aligned}
$$

The functions (48)-(50) can be viewed as a coordinate transformation and $u, v$ and $\tilde{P}$ as new coordinates on $\Gamma_{E}$. This is an interpretation of $\Phi$ that avoids the following paradox: $\tilde{P}_{-}$is to be totally undetermined on $\Gamma_{E}$ (see the previous section) and at the same time $\tilde{P}_{-}$has the same physical meaning as $\tilde{P}$, which is to be a well-defined function on $\Gamma_{E}$ by Eqs. (48)-(50). By considering Eq. (50) as a coordinate transformation, we regard $\tilde{P}$ as a coordinate on the right subspacetime, because $\tilde{P}_{+}$and $\tilde{P}$ are in one-to-one relation [see also Eq. (52) and (53)] and $\tilde{P}_{+}$is such a coordinate.

Another important point is that there is an infinity of different maps that satisfy above requirements, if one exists. This follows from symmetry: given a map $\Phi$, we can sandwich it between the symmetries to obtain another one. The non-uniqueness of $\Phi$, however, leads to an uncertainty in the coordinate $\tilde{P}_{+}$(and so to that of the Schwarzschild coordinate $T_{+}$) of the shell. The symmetry $G$ is, from the point of view of the spacetime geometry of each shell spacetime, nothing but a remainder of the original general covariance: the Schwarzschild or the Kruskal coordinates are not uniquely determined by the geometry of a fixed Kruskal spacetime. To map the dynamics of the system $\left(\Gamma_{1}, \Theta_{1}\right)$ to $\left(\Gamma_{E}, \Theta_{E}\right)$, a particular $\Phi$ must be fixed. Which one does not seem to matter as long as (classical) physical properties are concerned: a change in $\Phi$ amounts just to relabeling the classical dynamical trajectories.

In a quantum theory such a relabeling may lead to a problem, however. As an example, consider the unitary extension of the quantum shell dynamics described in Ref. [7]. There, one works with coordinates analogous to $u, v$ and $\tilde{P}$ and one uses the simplicity of the Hamiltonian in these coordinates to show the existence (if the rest mass of the shell is smaller than Planck mass) and uniqueness of a self-adjoint extension of the Hamiltonian. 
The eigenfunctions of the extended Hamiltonian are linear combinations of contracting and expanding waves. One can construct wave packets from them that are, at each time $T$, spacially concentrated around a well-defined wave-function maximum so that one can plot the radius $R_{M}$ of the maximum as a function of the time $T$. The function $R_{M}(T)$ diverges for $T \rightarrow-\infty$, then decreases till, at some $T_{0}$, a minimum $R_{M}\left(T_{0}\right)>0$ of $R_{M}(T)$ is reached, and then increases again to infinity as $T \rightarrow \infty$. For each wave packet one can define a time delay $\Delta T$ between the departure of the packet at $R=\infty$ and the arrival at $R=\infty$. Of course, $\Delta T$ is not the limit $\lim _{R=\infty}\left[T_{2}(R)-T_{1}(R)\right]$, where $T_{1,2}(R)$ are the two solutions of the equation $R_{M}(T)=R$, because the difference $T_{2}(R)-T_{1}(R)$ diverges as $R \rightarrow \infty$; one defines $\Delta T$ e.g. by comparing the packet 'trajectory' with some standard scattering trajectory.

To interpret this scattering, we need, however, the function $R_{M}\left(T_{+}\right)$rather than $R_{M}(T)$. In principle, we can calculate the first from the second by using some fixed map $\Phi$. The problem is that two different maps, $\Phi_{1}$ and $\Phi_{2}$, say, will then lead to two different $R_{M}\left(T_{+}\right)$that imply in turn two different time delays $\Delta T$. This is clear because contracting (expanding) part of $R_{M}(T)$ lies in the subset of the phase space that contains contracting (expanding) scattering trajectories and the difference between $\Phi_{1}$ and $\Phi_{2}$ along the expanding trajectories is independent from their difference along contracting trajectories.

\section{A. The differential equation for $\Phi$ in the general case}

In this subsection, we shall return to the classical theory and reformulate the above requirements on $\Phi$ in the form of a differential equation. We assume that $E>0$ and $E_{+}>0$; the special case $E=0$ is studied in the Appendix. We have two manifolds, $\Gamma_{1}$ with coordinates $u, v$, and $\tilde{P}$ that satisfy the conditions $R>0$ and $E_{+}>0$, and $\Gamma_{E}$ with the coordinates $u_{-}, v_{-}, \tilde{P}_{+}$satisfying the same condition (which is independent of $\tilde{P}_{-}$or $\tilde{P}_{+}$). According to the point 2,

$$
d\left(\Phi_{*} \Theta_{E}-\Theta_{1}\right)=0 .
$$


The functions $u$ and $u_{-}$as well as $v$ and $v_{-}$represent the same physical quantities, so according to the point 3 the transformation (48)-(50) has to preserve them:

$$
\begin{aligned}
& u_{-} \circ \Phi(u, v, \tilde{P})=u, \\
& v_{-} \circ \Phi(u, v, \tilde{P})=v
\end{aligned}
$$

Thus, the only non-trivial part of $\Phi$ is the transformation (50); let us denote the corresponding function by $\phi$ :

$$
\phi(u, v, \tilde{P}):=\tilde{P}_{+} \circ \Phi(u, v, \tilde{P}) .
$$

Substituting Eqs. (52)-(54) into Eq. (51), we obtain

$$
d\left(\left.\Theta_{E}\right|_{E_{-}=E, u_{-}=u, v_{-}=v, \tilde{P}_{+}=\phi}-\Theta_{1}\right)=0 .
$$

This is a partial differential equation of the first order for the function $\phi$; its characteristics are easily found to coincide with the curves $E_{+}=$const. We can, therefore, reduce this equation to an ordinary differential equation, if we transform it to the coordinates $R$ and $E_{+}$. That is our next task.

Let us define the functions $A, B, A^{\prime}$ and $B^{\prime}$ of $R$ and $E_{+}$by

$$
\begin{aligned}
\left.\Theta_{E}\right|_{E_{-}=E, u_{-}=u, v_{-}=v, \tilde{P}_{+}=\phi} & =A d R+B d E_{+}-4 E_{+} d \phi \\
\Theta_{1} & =A^{\prime} d R+B^{\prime} d E_{+}-4 E_{+} d \tilde{P} .
\end{aligned}
$$

Substituting Eqs. (56) and (57) into Eq. (55), we obtain the equations:

$$
\begin{aligned}
A_{E_{+}}-A_{E_{+}}^{\prime}-4 \phi_{R} & =B_{R}-B_{R}^{\prime}, \\
\phi_{\tilde{P}} & =1
\end{aligned}
$$

(the indices denote partial derivatives). These two equations are equivalent to the system

$$
\begin{aligned}
\phi & =\tilde{P}+\Delta\left(R, E_{+}\right), \\
\frac{\partial \Delta}{\partial R} & =\frac{1}{4}\left(A_{E_{+}}-B_{R}-A_{E_{+}}^{\prime}+B_{R}^{\prime}\right) .
\end{aligned}
$$


Eq. (59) is the desired ordinary differential equation (for the function $\Delta$ ).

Let us work out the explicit $R$ - $E_{+}$-dependence of the right-hand side. We obtain from Eq. (31) and (45):

$$
\begin{aligned}
A & =\left(\frac{R^{2}}{4}-E^{2}\right)\left(\ln \left|\frac{u}{v}\right|\right)_{R}-\left(\frac{R^{2}}{4}-E_{+}^{2}\right)\left(\ln \left|\frac{u_{+}}{v_{+}}\right|\right)_{R}, \\
B & =\left(\frac{R^{2}}{4}-E^{2}\right)\left(\ln \left|\frac{u}{v}\right|\right)_{E_{+}}-\left(\frac{R^{2}}{4}-E_{+}^{2}\right)\left(\ln \left|\frac{u_{+}}{v_{+}}\right|\right)_{E_{+}}, \\
A^{\prime} & =-\frac{E M^{2}}{R}\left(\ln \left|\frac{u}{v}\right|\right)_{R}-2 E M(u+v)_{R}-\frac{M}{2}(u+v)\left(1+\frac{2 E}{R}\right), \\
B^{\prime} & =-\frac{E M^{2}}{R}\left(\ln \left|\frac{u}{v}\right|\right)_{E_{+}}-2 E M(u+v)_{E_{+} .}
\end{aligned}
$$

We find easily

$$
\begin{aligned}
A_{E_{+}}-B_{R} & =2 E_{+}\left(\ln \left|\frac{u}{v}\right|\right)_{R}-\frac{R}{2}\left(\ln \left|\frac{u}{v}\right|\right)_{E_{+}}+\frac{R}{2}\left(\ln \left|\frac{u_{+}}{v_{+}}\right|\right)_{E_{+}}, \\
A_{E_{+}}^{\prime}-B_{R}^{\prime} & =E\left(\frac{M^{2}}{R}\right)_{R}\left(\ln \left|\frac{u}{v}\right|\right)_{E_{+}}+2 E M_{R}(u+v)_{E_{+}} \\
& -\frac{M}{2}\left(1+\frac{2 E}{R}\right)(u+v)_{E_{+} .}
\end{aligned}
$$

Next, we have to express everything in terms of $R$ and $E_{+}$, using Eqs. (32) and (33). As the first step, we derive the following helpful equations:

$$
\begin{gathered}
u=-\frac{E_{+}-E}{M}-\frac{M}{2 R}+\omega \frac{\sqrt{\mathcal{P}}}{M R}, \\
v=\frac{E_{+}-E}{M}+\frac{M}{2 R}+\omega \frac{\sqrt{\mathcal{P}}}{M R}, \\
u_{+}=-\frac{E_{+}-E}{M}+\frac{M}{2 R}+\omega \frac{\sqrt{\mathcal{P}}}{M R}, \\
v_{+}=\frac{E_{+}-E}{M}-\frac{M}{2 R}+\omega \frac{\sqrt{\mathcal{P}}}{M R},
\end{gathered}
$$

where

$$
\mathcal{P}(R):=-M^{2}(R) R^{2} V(R) .
$$

A straightforward calculation then gives 


$$
\begin{aligned}
A_{E_{+}}-B_{R}= & -\frac{\omega}{\sqrt{\mathcal{P}}}\left(R+2 E_{+}\right)\left(E_{+}-E+\frac{M^{2}}{2 R}\right)+ \\
& \frac{\omega M_{R}}{M \sqrt{\mathcal{P}}}\left(\left(4 E_{+}^{2}-4 E_{+} E\right) R+2 M^{2} E_{+}\right) \\
A_{E_{+}}^{\prime}-B_{R}^{\prime}= & -\frac{\omega}{2 R \sqrt{\mathcal{P}}}\left(2\left(E_{+}-E\right) R^{2}+\left(4 E_{+} E-4 E^{2}+M^{2}\right) R-2 E M^{2}\right)+ \\
& \omega \frac{2 E M_{R}}{M \sqrt{\mathcal{P}}}\left(2\left(E_{+}-E\right) R-M^{2}\right) .
\end{aligned}
$$

Collecting all pieces, we arrive at the differential equation for $\Delta$ in the form

$$
\begin{aligned}
\frac{d \Delta}{d R} & =\frac{\omega}{4 \sqrt{\mathcal{P}}}\left(-2\left(E_{+}-E\right)^{2}+M^{2}\left(E_{+}+E\right) \frac{1}{R}\right) \\
& +\omega \frac{M_{R}}{M \sqrt{\mathcal{P}}}\left(\left(E_{+}-E\right)^{2} R+M^{2}\left(E_{+}+E\right)\right) .
\end{aligned}
$$

Observe that the upper and lower $u$-v-plane give the same curves, only their orientation is different.

\section{B. Solution of the equation for $\Delta$ in the case of dust}

In the general case, we cannot say much about the solution of Eq. (60), because the function $M(R)$ in it is an arbitrary (positive) function. In the case of dust, $M=$ const, however, the equation is readily solvable. In this case, we obtain

$$
\frac{d \Delta}{d R}=\frac{\omega}{4 \sqrt{\mathcal{P}}}\left(-2\left(E_{+}-E\right)^{2}+M^{2}\left(E_{+}+E\right) \frac{1}{R}\right)
$$

Eq. (61) can be solved by elementary integration; the solution, however, will change its form if the parameters $E_{+}, E$ and $M$ vary. Thus, we obtain only a local form of the function $\Delta$. In the present subsection, a careful discussion of the different cases is given. The results of this discussion will be used in the next subsection where we try to match the different cases smoothly together.

Let us write the solution of Eq. (61) in each of the half $u$-v-plane, $\omega= \pm 1$, as follows:

$$
\Delta^{\omega}=\Delta_{0}^{\omega}+\Delta_{1}^{\omega}+\Delta_{2}^{\omega}
$$

where $\Delta_{0}^{\omega}=\Delta_{0}^{\omega}\left(E_{+}\right)$is an arbitrary function of $E_{+}$(integration constant - this is the nonuniqueness in $\Phi$ due to the symmetry), 


$$
\Delta_{1}^{\omega}:=-\omega \frac{\left(E_{+}-E\right)^{2}}{2} \int \frac{d R}{\sqrt{\mathcal{P}}}
$$

and

$$
\Delta_{2}^{\omega}:=\omega \frac{M^{2}\left(E_{+}+E\right)}{4} \int \frac{d R}{R \sqrt{\mathcal{P}}}
$$

For dust, the function $\mathcal{P}$ becomes a quadratic polynomial of $R$ :

$$
\mathcal{P}(R):=\left(\left(E_{+}-E\right)^{2}-M^{2}\right) R^{2}+\left(E_{+}+E\right) M^{2} R+\frac{M^{4}}{4},
$$

The discriminant of the quadratic equation $\mathcal{P}(R)=0$ equals to $M^{4}\left(4 E E_{+}+M^{2}\right)$; it is always positive, so there are two roots:

$$
R_{1}=-\frac{M^{2}}{2} \frac{1}{\left(E_{+}+E\right)+\sqrt{4 E_{+} E+M^{2}}}<0
$$

and

$$
R_{2}=-\frac{M^{2}}{2} \frac{\left(E_{+}+E\right)+\sqrt{4 E_{+} E+M^{2}}}{\left(E_{+}-E\right)^{2}-M^{2}} .
$$

$R_{2}$ is positive for $\left(E_{+}-E\right)^{2}-M^{2}<0$, that is $E_{+} \in(\operatorname{Max}(0, E-M), E+M)$. Then $\mathcal{P}(R)>0$ in the interval $R \in\left(0, R_{2}\right)$ and the trajectory is bound, with maximal radius $R_{2}$. For $\left(E_{+}-E\right)^{2}-M^{2} \geq 0, R_{2}<R_{1}<0, \mathcal{P}(R)>0$ in the interval $R \in(0, \infty)$ and the trajectory is unbound.

To calculate the function $\Delta_{1}^{\omega}$, we have to distinguish three cases.

$\underline{\left(E_{+}-E\right)^{2}-M^{2}>0}$. In terms of the variables $E, E_{+}$and $M$, this means that either $E_{+}>E+M$ or $E_{+}<E-M$. The corresponding trajectories are the contracting and expanding scattering states. Then

$$
\Delta_{1}^{\omega}\left(R, E_{+}\right)=-\omega \frac{\left(E_{+}-E\right)^{2}}{2 \sqrt{\alpha}} \operatorname{arccosh} \frac{2 \alpha R+M^{2}\left(E_{+}+E\right)}{M^{2} \sqrt{4 E_{+} E+M^{2}}}
$$

where $\alpha:=\left(E_{+}-E\right)^{2}-M^{2}$; we use the increasing branch of arccosh. $\Delta_{1}^{\omega}$ is regular for all $R \in(0, \infty)$. The values at the end-points are:

$$
\Delta_{1}^{\omega}\left(0, E_{+}\right)=-\omega \frac{\left(E_{+}-E\right)^{2}}{2 \sqrt{\alpha}} \operatorname{arccosh} \frac{E_{+}+E}{\sqrt{4 E_{+} E+M^{2}}}
$$


and $\Delta_{1}^{\omega}$ diverges logarithmically with $R \rightarrow \infty$ to $-\omega \infty$.

$\underline{\left(E_{+}-E\right)^{2}-M^{2}=0 .}$. Then $R_{1}<0, R_{2} \rightarrow \infty$ and the polynomial $\mathcal{P}(R)>0$ for all $R \in$ $(0, \infty)$. The trajectories are the parabolic scattering states separating the scattering states from the bound states. The integral is:

$$
\Delta_{1}^{\omega}=-\omega \frac{\left(E_{+}-E\right)^{2}}{2\left(E_{+}+E\right)} \sqrt{4\left(E_{+}+E\right) R+M^{2}} .
$$

It is again regular for all $R \in(0, \infty)$. The values at the end-points are:

$$
\Delta_{1}^{\omega}\left(0, E_{+}\right)=-\omega \frac{\left(E_{+}-E\right)^{2} M}{2\left(E_{+}+E\right)}
$$

and it diverges as $R^{1 / 2}$ for $R \rightarrow \infty$.

$\underline{\left(E_{+}-E\right)^{2}-M^{2}<0}$. In terms of the variables $E, E_{+}$and $M$, this means that $E_{+} \in$ $(\operatorname{Max}(0, E-M), E+M)$. Then $R_{1}<0<R_{2}$. The corresponding trajectories are the bound states and the integral is

$$
\Delta_{1}^{\omega}\left(R, E_{+}\right)=-\omega \frac{\left(E_{+}-E\right)^{2}}{2 \sqrt{-\alpha}} \arccos \frac{2 \alpha R+M^{2}\left(E_{+}+E\right)}{M^{2} \sqrt{4 E_{+} E+M^{2}}}
$$

(we use the decreasing branch of arccos). $\Delta_{1}^{\omega}$ is regular for all $R \in\left(0, R_{2}\right)$. The values at the endpoints are

$$
\Delta_{1}^{\omega}\left(0, E_{+}\right)=-\omega \frac{\left(E_{+}-E\right)^{2}}{2 \sqrt{-\alpha}} \arccos \frac{E_{+}+E}{\sqrt{4 E_{+} E+M^{2}}}
$$

and

$$
\Delta_{1}^{\omega}\left(R_{2}, E_{+}\right)=-\omega \frac{\left(E_{+}-E\right)^{2}}{2 \sqrt{-\alpha}} \pi
$$

The function $\Delta_{2}^{\omega}$ can be calculated immediately with the result

$$
\Delta_{2}^{\omega}\left(R, E_{+}\right)=-\omega \frac{E_{+}+E}{2} \ln \frac{2\left(E_{+}+E\right) R+M^{2}+2 \sqrt{\mathcal{P}}}{2 R \sqrt{4 E_{+} E+M^{2}}},
$$

which is valid for all values of $E_{+}, E, M$, and $R$ for which $\mathcal{P}>0 . \Delta_{2}^{\omega}$ diverges at $R \rightarrow 0$ for all trajectories. For scattering trajectories,

$$
\Delta_{2}^{\omega}\left(\infty, E_{+}\right)=-\omega \frac{E_{+}+E}{2} \ln \frac{\left(E_{+}+E\right)+\sqrt{\alpha}}{\sqrt{4 E_{+} E+M^{2}}}
$$


is finite. For bound trajectories,

$$
\Delta_{2}^{\omega}\left(R_{2}, E_{+}\right)=-\omega \frac{E_{+}+E}{2} \ln \frac{\left(E_{+}+E\right)+\sqrt{4 E_{+} E+M^{2}}}{2}
$$

is also finite and for the limit $R_{2} \rightarrow \infty\left(E_{+} \rightarrow E \pm M\right)$, we obtain

$$
\lim _{R_{2}=\infty} \Delta_{2}^{\omega}\left(R_{2}, E_{+}\right)=-\omega \frac{2 E \pm M}{2} \ln (2 E \pm M)
$$

\section{Matching and patching}

The function $\Delta\left(R, E_{+}\right)$must be well-defined and smooth for all values $E_{+}>0$ and $R>0$ of its variables, and this at all values $E>0$ and $M>0$ of its parameters in order that it determines a map $\Phi$ with the required properties. The 'pieces' of $\Delta$ obtained in the previous section must, therefore, be smoothly matched together. In the present subsection we prove that this is impossible and give some discussion of the negative result.

Within the half planes $u+v<0(\omega<0)$ and $u+v>0(\omega>0)$, the function $\Delta_{2}^{\omega}\left(R, E_{+}\right)$is smooth everywhere, but $\Delta_{1}^{\omega}$ as a function of $E_{+}$seems to be divergent at $\left(E_{+}-E\right)^{2}-M^{2}=0$. A routine inspection in the complex plane reveals that $\Delta_{1}^{\omega}$ is, in fact, a smooth function there for each $\omega$. Hence, the sum $\Delta_{1}^{\omega}\left(R, E_{+}\right)+\Delta_{2}^{\omega}\left(R, E_{+}\right)$is smooth inside each half plane. The whole function $\Delta^{\omega}$ can, therefore, be made smooth by an arbitrary smooth choice of $\Delta_{0}^{\omega}$

The main problem is the matching at the boundary $u+v=0$ between the two half planes. Let us first study some properties of the curves $E_{+}=$const in the $u$ - $v$-plane. The Kruskal coordinates $U$ and $V$ are both future oriented; hence the past singularity $R=0$ lies in the quadrant $U<0, V<0$, and the future one lies in the quadrant $U>0$, $V>0$. The transformation (25) [or (35)] preserves signs, so the past singularity lies in the lower, and the future one in the upper half of the $u$-v-plane. Thus, in the lower half plane $(u+v<0), R$ is increasing along all trajectories and in the upper half plane $(u+v>0)$, $R$ is decreasing. Observe that only bound trajectories can cross over from one half plane to 
the other; scattering trajectories are always imprisoned inside one half plane: the expanding $(\dot{R}>0)$ in the lower and the contracting $(\dot{R}<0)$ in the upper half plane.

Let us try to extend $\Delta$ continuously across the boundary $u+v=0$ using the remaining freedom in $\Delta_{0}^{\omega}\left(E_{+}\right)$. We shall split $\Delta_{0}^{\omega}\left(E_{+}\right)$into two terms, $\Delta_{01}^{\omega}\left(E_{+}\right)+\Delta_{02}^{\omega}\left(E_{+}\right)$. In the lower half plane, $\Delta_{1}^{-}$is increasing with $R$ if $E_{+}$is kept constant. If $E_{+} \in(\operatorname{Max}(0, E-M), E+M)$, $\Delta_{1}^{-}$assumes the value (66) for $\omega=-1$ at the boundary $u+v=0$. In the upper half plane $\Delta_{1}^{+}$is decreasing with $R$ and it reaches the boundary with the value (66) for $\omega=+1$. Thus, we can construct a continuous function by choosing $\Delta_{01}^{-}\left(E_{+}\right)=0$ and

$$
\Delta_{01}^{+}\left(E_{+}\right)=\frac{\left(E_{+}-E\right)^{2}}{\sqrt{-\alpha}} \pi .
$$

The function $\Delta_{1}^{+}\left(R, E_{+}\right)+\Delta_{01}^{+}\left(E_{+}\right)$in the upper half plane, together with $\Delta_{1}^{-}\left(R, E_{+}\right)$in the lower, define in fact a smooth function at all points the lower half plane, at the boundary $u+v=0$, and in the subset $E_{+} \in(\operatorname{Max}(0, E-M), E+M)$ of the upper half plane; let us denote this set by $\mathcal{D}_{-}$. $\mathcal{D}_{-}$consists of all points at the expanding scattering and at the bound trajectories. However, there is no continuous extension of this function to the rest of the upper half plane, because it diverges at the points of the upper half plane satisfying $\left(E_{+}-E\right)^{2}-M^{2}=0$, that is, at the contracting parabolic trajectories.

Let us turn to the function $\Delta_{2}^{-}\left(R, E_{+}\right)$starting again in the lower half plane. It is increasing from the value $-\infty$ at $R=0$ to a finite value at $R=\infty$ along all scattering trajectories, and to a finite value (68) with $\omega=-1$ along bound trajectories at the boundary $u+v=0$. The function (68) has finite limits as $E_{+}$approaches the values $E \pm M$. Thus, there is no problem to extend this $\Delta_{2}^{-}\left(R, E_{+}\right)$to the whole upper half plane. One has just to choose $\Delta_{02}^{-}\left(E_{+}\right)=0$ and

$$
\Delta_{02}^{+}\left(E_{+}\right)=\left(E_{+}+E\right) \ln \frac{E_{+}+E+\sqrt{4 E_{+} E+M^{2}}}{2}
$$

everywhere. The two functions $\Delta_{2}^{-}\left(R, E_{+}\right)$in the lower and $\Delta_{2}^{+}\left(R, E_{+}\right)+\Delta_{02}^{+}\left(E_{+}\right)$in the upper half plane form together a smooth function in the whole $u$-v-plane.

It is also clear that no continuous choice of $\Delta_{01}^{-}+\Delta_{02}^{-}$can remove the singularity in the upper half plane. Hence, any allowed choice of $\Delta$ that is continuous in the lower half plane 
will necessarily diverge at all points of the contracting parabolic trajectories $\left(E_{+}=E \pm M\right)$ in the upper half plane. This result already means that there is no function $\Delta$ that would be continuous everywhere.

An analogous construction starting from the upper half plane leads to analogous results: $\Delta_{1}$ can be made smooth only in the open subset $\mathcal{D}_{+}$of the $u$-v-plane that contains points of all contracting scattering and all bound trajectories. It diverges at all expanding parabolic trajectories. $\Delta_{2}$ can again be chosen smooth everywhere. Of course, the two constructions end up with two different solutions: in $\mathcal{D}_{-} \cap \mathcal{D}_{+}$, they differ by a function of $E_{+}$that diverges at all points satisfying $E_{+}=E \pm M$.

The two constructions in the foregoing paragraphs deliver, in fact, two maximal continuous extensions of our local solutions for $\Delta$. This two maximal continuous extensions are of course also smooth (if $\Delta_{0}^{\omega}$ are chosen so), but it is the continuity that is lost at the boundary. Similar conclusions can be drawn for the case $E=0$ or $E_{+}=0$, but the proof must use different vartiables, because $u$ and $v$ can serve as two regular coordinates only for $E>0$. The proof for the special case $E=0$ is sketched in the Appendix.

As discussed at the beginning of the present section, the map $\Phi$ (if it existed) can be considered as equivalence map (morphism) between the two systems $\left(\Gamma_{1}, \Theta_{1}\right)$ and $\left(\Gamma_{E}, \Theta_{E}\right)$ or, alternatively, as a definition of new coordinates, $u, v$, and $\tilde{P}$, for the system $\left(\Gamma_{E}, \Theta_{E}\right)$. The fact that $\Phi$ exists only locally and is non-unique suggests another interpretation: it is a pasting map between different patches of a larger presymplectic manifold. Let us describe an example of such a construction and see, whether it can be of any use or not.

We shall denote the two maximal extensions of $\Phi$ defined above with $\Delta_{0}^{-}=0$ or $\Delta_{0}^{+}=0$ by $\Phi_{-}$and $\Phi_{+}$, respectively; their domains in $\Gamma_{1}$ are $\mathcal{D}_{-}$and $\mathcal{D}_{+}$f. Consider two copies of

\footnotetext{
${ }^{5}$ Observe that the maximal extensions can be chosen differently, overlapping again at the bound trajectories, but the new domain $\mathcal{D}_{-}^{\prime}$ containing points of all scattering trajectories expanding to the right or contracting from the left and the new $\mathcal{D}_{+}^{\prime}$ containing points of all scattering trajectories
} 
$\left(\Gamma_{E}, \Theta_{E}\right)$ denoted by $\left(\Gamma_{E}^{\prime}, \Theta_{E}^{\prime}\right)$ and $\left(\Gamma_{E}^{\prime \prime}, \Theta_{E}^{\prime \prime}\right)$, and one copy of $\left(\Gamma_{1}, \Theta_{1}\right)$; let $\Phi_{+}^{\prime}: \Gamma_{1} \mapsto \Gamma_{E}^{\prime}$ has the domain $\mathcal{D}_{+} \subset \Gamma_{1}$ and let $\Phi_{-}^{\prime \prime}: \Gamma_{1} \mapsto \Gamma_{E}^{\prime \prime}$ has the domain $\mathcal{D}_{-} \subset \Gamma_{1}$. Let us paste $\Gamma_{1}$ and $\Gamma_{E}^{\prime}$ together along $\mathcal{D}_{+}$and $\Phi_{+}^{\prime}\left(\mathcal{D}_{+}\right)$by $\Phi_{+}^{\prime}$ and similarly $\Gamma_{1}$ and $\Gamma_{E}^{\prime \prime}$ along $\mathcal{D}_{-}$and $\Phi_{-}^{\prime \prime}\left(\mathcal{D}_{-}\right)$ by $\Phi_{-}^{\prime \prime}$. The result is a well-defined (possibly non-Hausdorff) presymplectic manifold, which will be denoted by $(\Gamma, \Theta)$.

Problems arise, however, if one tries to find a physical interpretation of this construction and considers the role and position of observers. An idea, which seems reasonable, is that each dynamical system like $\Gamma_{E}$ or $\Gamma_{1}$ is to describe, in an idealized manner, what a family of communicating observers can do with the shell. They can send it contracting in different dynamical states, observe its motion and also observe different states of expanding shells. It seems further reasonable that such observers could be placed somewhere in the right asymptotic regions of $\mathcal{M}_{+}$and $\mathcal{M}_{-}$(or left, but not both right and left, because right observers cannot communicate with the left ones). Indeed, the observers are in $\mathcal{M}_{-}$before they throw in a shell contracting from right, and then they are in $\mathcal{M}_{+}$; analogously for shells expanding to right, the observers are first in $\mathcal{M}_{+}$and, after the shell passes, in $\mathcal{M}_{-}$. In this sense, the right asymptotic regions of all spacetime shell solutions of one system are to be considered as identical.

Next consider the pasting of three systems $\Gamma_{E}^{\prime}, \Gamma_{1}$ and $\Gamma_{E}^{\prime \prime}$. Nothing seems to hinder us in making the assumption that each of the three dynamical systems has its own observer family of the above kind before the pasting. It is, therefore, conceivable that some of the three families remain distinct after the pasting. Then the observers of $\Gamma_{E}^{\prime}$ might send in a shell and this shell disappears behind a horizon for these observers, but appears during its motion somewhere else, where it can be observed by another of the three families. Let us look to see what happens with the families if we perform the pasting.

It is a pasting of phase spaces; but such a pasting implies also that points of spacetimes that expand to the left or that contract from the right. 
will be identified. Indeed, let us chose any point $\left(u_{-}^{\prime}, v_{-}^{\prime}, \tilde{P}_{+}^{\prime}\right) \in \Gamma_{E}^{\prime}$ that lies at a bound trajectory. $\left(u_{-}^{\prime}, v_{-}^{\prime}, \tilde{P}_{+}^{\prime}\right)$ determines via Eqs. (36) and (39) the Schwarzschild mass of the right subspacetime $\mathcal{M}_{+}^{\prime}$, via Eqs. (37), (38), (43) and (35) a point $\left(U_{+}^{\prime}, V_{+}^{\prime}\right)$ of $\mathcal{M}_{+}^{\prime}$, where the shell is, as well as the four-velocity of the shell at $\left(U_{+}^{\prime}, V_{+}^{\prime}\right)$ represented by $P_{K}^{\prime+}$ through Eq. (11). By $\Phi_{+}^{\prime-1}$, the point $\left(u_{-}^{\prime}, v_{-}^{\prime}, \tilde{P}_{+}^{\prime}\right)$ is identified with $(u, v, \tilde{P}) \in \Gamma_{1}$ and this, in turn by $\Phi_{-}^{\prime \prime}$ with the point $\left(u_{-}^{\prime \prime}, v_{-}^{\prime \prime}, \tilde{P}_{+}^{\prime \prime}\right) \in \Gamma_{E}^{\prime \prime}$. Again, $\left(u_{-}^{\prime \prime}, v_{-}^{\prime \prime}, \tilde{P}_{+}^{\prime \prime}\right)$ determines the mass $E_{+}^{\prime \prime}=E_{+}^{\prime}$ of $\mathcal{M}_{+}^{\prime \prime}$, the point $\left(U_{+}^{\prime \prime}, V_{+}^{\prime \prime}\right)$ of $\mathcal{M}_{+}^{\prime \prime}$ and a four-velocity $P_{K}^{\prime \prime+}$ at $\left(U_{+}^{\prime \prime}, V_{+}^{\prime \prime}\right)$.

It seems, therefore, that the points $\left(U_{+}^{\prime}, V_{+}^{\prime}\right)$ and $\left(U_{+}^{\prime \prime}, V_{+}^{\prime \prime}\right)$ must be identical after the pasting, because they lie at the trajectory of one and the same shell. In fact, one can easily see that all points of the trajectory then lie in these particular spacetimes $\mathcal{M}_{+}^{\prime}$ and $\mathcal{M}_{+}^{\prime \prime}$ and must be identical in pairs. Thus, $\mathcal{M}_{+}^{\prime}$ and $\mathcal{M}_{+}^{\prime \prime}$ have a one-dimensional set of points in common. This is very strange unless the spacetimes $\mathcal{M}_{+}^{\prime}$ and $\mathcal{M}_{+}^{\prime \prime}$ are themselves identical. In fact, there is only one isometry mapping $\mathcal{M}_{+}^{\prime}$ onto $\mathcal{M}_{+}^{\prime \prime}$ and preserving the trajectory points. It is natural to assume that such an identification is performed in all cases where the above construction is viable; that is, $\mathcal{M}_{+}^{\prime}$ and $\mathcal{M}_{+}^{\prime \prime}$ are identical for all $E_{+}^{\prime}=E_{+}^{\prime \prime} \in\left(E_{-}-M, E_{-}+M\right)$. Notice that there is no analogous argument for $\mathcal{M}_{-}^{\prime}$ and $\mathcal{M}_{-}^{\prime \prime}$, because a point of $\Gamma_{E}^{\prime}\left(\right.$ or $\left.\Gamma_{E}^{\prime \prime}\right)$ does not determine a unique point of $\mathcal{M}_{+}^{\prime}\left(\right.$ or $\left.\mathcal{M}_{+}^{\prime \prime}\right)$.

It seems to follow that the pasting leads to identification of all three families of observers. Indeed, if the observers of the system $\Gamma_{E}^{\prime}$ throw in a shell in a bound state (remember that there are bound states passing through any radius, and, moreover, 'lower' bound shells can in any case be arraged indirectly by the asymptotic observers) the same must be done at the same time and radius by each of the other families. This is 'disappointing' but not desastrous. However, if the observers of the system $\Gamma_{E}^{\prime}$ throw in a shell in a scattering state, then the same must be done by the observers of $\Gamma_{1}$, but it must not be done by the observers of $\Gamma_{E}^{\prime \prime}$, because contracting scattering states of $\Gamma_{E}^{\prime}$ are distinct from those of $\Gamma_{E}^{\prime \prime}$ according to the pasting procedure. This seem to be a paradox; I have not found any way out of it as yet. Thus, the pasting does not seem to work. 
To summarize: The map $\Phi$ that realizes the equivalence between the systems $\left(\Gamma_{1}, \Theta_{1}\right)$ and $\left(\Gamma_{E}, \Theta_{E}\right)$ is afflicted with two problems. On one hand, a map satisfying all requirements 1-3 at the beginning of Sec. $\nabla$ does not exist, at least for the case of dust. One might be able to weaken the continuity in some cautious way and still preserve the physical content, but this is only a speculation that must be studied. On the other hand, the map $\Phi$ is not unique; this does not seem to lead to any serious difficulty as long as just the classical theory is concerned, but it can be a handicap for the self-adjoint extension methods in the quantum theory.

\section{Acknowledgements}

Discussions with K. Kuchař and G. Lavrelashvili are thankfully acknowledged.

\section{APPENDIX}

In this Appendix, we shall sketch the main steps of the line of reasoning in terms of Schwarzschild coordinates so that the important special case $E_{-}=0$ of flat shell interior can be dealth with.

The Warsaw approach starts in this case from the action of the form

$$
S_{1}=\int d t\left(p_{T} \dot{T}+p_{R} \dot{R}-\mathcal{N C}_{1}\right)
$$

where $T$ and $R$ are coordinates on a two-dimensional Minkowski half-spacetime $R>0$ and

$$
\mathcal{C}_{1}=-\frac{1}{2}\left(-p_{T}+\frac{M^{2}(R)}{2 R}\right)^{2}+\frac{1}{2} p_{R}^{2}+\frac{1}{2} M^{2}(R) .
$$

In order to reduce this action to the Cartan form, we introduce the Schwarzschild (here: Minkowski) momentum $P_{S}$ by

$$
P_{S}:=R \operatorname{arctanh} \frac{d R}{d T}
$$

The constraint $\mathcal{C}_{1}=0$ is then identically satisfied by 


$$
\begin{aligned}
& p_{T}=-M(R) \cosh \frac{P_{S}}{R}+\frac{M^{2}(R)}{2 R}, \\
& p_{R}=M(R) \sinh \frac{P_{S}}{R}
\end{aligned}
$$

and the Cartan form becomes

$$
\Theta_{1}=-M(R)\left(\cosh \frac{P_{S}}{R}-\frac{M(R)}{2 R}\right) d T+M(R) \sinh \frac{P_{S}}{R} d R
$$

The Potsdam approach in the Schwarzschild coordinates (Ref. [11]) is awkward, because there are 16 disjoint ranges of validity of these coordinates (4 quadrants for each Kruskal subspacetime). We shall need four sign functions $a_{\epsilon}$ and $b_{\epsilon}, \epsilon= \pm 1$ and an abbreviation $\operatorname{sh}_{a} x$ in order that we can catch all 16 quadrants by a single formula. The definitions are: $a_{\epsilon}:=\operatorname{sgn} F_{\epsilon}$, where $F_{\epsilon}:=1-2 E_{\epsilon} / R, b_{\epsilon}:=+1$ in the past and $b_{\epsilon}:=-1$ in the future of the Kruskal event horizon in $\mathcal{M}_{\epsilon}$ and

$$
\operatorname{sh}_{a} x:=\frac{\mathrm{e}^{x}+a \mathrm{e}^{-x}}{2}
$$

The momentum $P_{S}^{\epsilon}$ conjugate to $R_{\epsilon}$ is determined by the 'Schwarzschild velocity' $d R / d T$ of the shell as follows (cf. [11]):

$$
P_{S}^{\epsilon}:=R_{\epsilon} \operatorname{arctanh}\left(\frac{1}{F_{\epsilon}} \frac{d R_{\epsilon}}{d T_{\epsilon}}\right)^{a_{\epsilon}}
$$

where $d R_{\epsilon} / d T_{\epsilon}$ is the derivative of the Schwarzschild radius $R_{\epsilon}$ with respect to the Schwarzschild time $T_{\epsilon}$ along the shell from the $\epsilon$-side. The action reads

$$
S_{2}=\int d t\left(\left[P_{S} \dot{R}-E \dot{T}\right]+\tilde{\nu}\left(R_{+}-R_{-}\right)-\nu \mathcal{C}_{2}\right)
$$

where $\tilde{\nu}$ and $\nu$ are Lagrange multipliers and

$$
\mathcal{C}_{2}:=\left[b R \sqrt{|F|} \operatorname{sh}_{a} \frac{P_{S}}{R}\right]+M(\bar{R})
$$

There is a secondary constraint, $\chi=0$, where

$$
\chi:=\left(\frac{\partial \mathcal{C}_{2}}{\partial \bar{P}_{S}}\right)_{\left[P_{S}\right]}
$$


explicitly,

$$
\chi=\left[b \sqrt{|F|} \operatorname{sh}_{-a} \frac{P_{S}}{R}\right] .
$$

The three constraints $\mathcal{C}_{2}=0, R_{+}=R_{-}$and $\chi=0$ can be rewritten as the following system of equations for $R_{+}, E_{+}, P_{+} a_{+}$and $b_{+}$:

$$
\begin{gathered}
R_{+}=R, \quad a_{+}=\operatorname{sgn} F_{+} \\
b_{+} \sqrt{\left|F_{+}\right|} \operatorname{sh}_{a_{+}} \frac{P_{S}^{+}}{R}=\cosh \frac{P_{S}^{-}}{R}-\frac{M(R)}{R}, \\
b_{+} \sqrt{\left|F_{+}\right|} \operatorname{sh}_{-a_{+}} \frac{P_{S}^{+}}{R}=\sinh \frac{P_{S}^{-}}{R}
\end{gathered}
$$

we have left out the index "-" and we have already set $E=0, a_{-}=+1, b_{-}=+1$ and $F_{-}=1$ everywhere. It follows immediately that

$$
F_{+}=\left(\cosh \frac{P_{S}^{-}}{R}-\frac{M(R)}{R}\right)^{2}-\sinh ^{2} \frac{P_{S}^{-}}{R},
$$

and

$$
\tanh \frac{P_{S}^{+}}{R}=\left(\frac{\sinh \frac{P_{S}^{-}}{R}}{\cosh \frac{P_{S}^{-}}{R}-\frac{M(R)}{R}}\right)^{a_{+}} .
$$

Eq. (76) can be rewritten in the form

$$
F_{+}=Q_{1} Q_{2}
$$

where

$$
\begin{aligned}
& Q_{1}:=1-\frac{M(R)}{R} \mathrm{e}^{-P_{S}^{-} / R}, \\
& Q_{2}:=1-\frac{M\left(R_{S}\right)}{R} \mathrm{e}^{P_{S}^{-} / R},
\end{aligned}
$$

are two important abbreviations. We also define the following sign functions:

$$
q_{1}:=\operatorname{sgn} Q_{1}, \quad q_{2}:=\operatorname{sgn} Q_{2} .
$$


Then, Eq. (78) implies

$$
a_{+}=q_{1} q_{2}
$$

and

$$
E_{+}=\frac{R}{2}\left(1-Q_{1} Q_{2}\right)
$$

Explicitly,

$$
E_{+}=E_{-}+M(R) \cosh \frac{P_{S}^{-}}{R}-\frac{M^{2}(R)}{2 R} .
$$

Let us turn to Eq. (77). We use the following simple identity

$$
\operatorname{arctanh} x^{a}=\frac{1}{2} \ln \left(a \frac{1+x}{1-x}\right)
$$

that holds for $a= \pm 1$ and all $|x|<1$. It implies that

$$
P_{S}^{+}=\frac{R}{2} \ln \left(a_{+} \frac{Q_{1}}{Q_{2}}\right)+P_{S}^{-} .
$$

However,

$$
a_{+} \frac{Q_{1}}{Q_{2}}=\left|\frac{Q_{1}}{Q_{2}}\right|
$$

because of Eq. (81) so that

$$
P_{S}^{+}=\frac{R}{2} \ln \left|\frac{Q_{1}}{Q_{2}}\right|+P_{S}^{-}
$$

or equivalently

$$
\mathrm{e}^{P_{S}^{+} / R}=\sqrt{\left|\frac{Q_{1}}{Q_{2}}\right|} \mathrm{e}^{P_{S}^{-} / R} .
$$

As for $b_{+}$, we just substitute Eq. (83) for $\mathrm{e}^{ \pm P_{S}^{+} / R}$ and Eq. (78) for $F_{+}$into the equation $\chi=0$; this leads to

$$
b_{+}\left(\left|Q_{1}\right| \mathrm{e}^{P_{S}^{-} / R}-a_{+}\left|Q_{2}\right| \mathrm{e}^{-P_{S}^{-} / R}\right)=2 \sinh \frac{P_{S}^{-}}{R}
$$


Using the definitions of $q_{1}, q_{2}, Q_{1}$ and $Q_{2}$, we obtain easily

$$
b_{+}=q_{1} .
$$

From Eqs. (81) and (84), it follows that $Q_{1}$ changes sign at the "black hole" and $Q_{2}$ at the "white hole" horizon of $\mathcal{M}_{+}$.

Subsituting Eq. (82) for $E_{+}$, Eq. (83) for $P_{S}^{+}$and $R$ for $R_{+}$and $R_{-}$into the variational principle (69), we obtain the reduced action with the Cartan form $\Theta_{E}$ given by

$$
\Theta_{2}:=\frac{R}{2} \ln \left|\frac{Q_{1}}{Q_{2}}\right| d R-M(R)\left(\cosh \frac{P_{S}^{-}}{R}-\frac{M(R)}{2 R}\right) d T_{+} .
$$

The cyclic coordinate $T_{-}$is automatically excluded, because it is contained only in one term of the form $E_{-} d T_{-}$and $E_{-}=0$. The manifold $\Gamma_{E}$ is determined by the inequalities

$$
R>0, \quad R \neq 2 E_{+}, \quad E_{+} \geq 0,
$$

that must be satisfied by the coordinates $R, P_{S}^{-}$, and $T_{+}$; it consists of four open disjoint submanifolds.

Finally, we have to show that there is a transformation

$$
T_{+}=\tilde{\phi}\left(T, R, P_{S}\right)
$$

such that $\Theta_{E}$ becomes to $\Theta_{1}$ plus possibly a closed form, if we substitute $\tilde{\phi}$ for $T_{+}$. In an analogous way as in Sec. VA, we obtain

$$
\tilde{\phi}\left(T, R, P_{S}\right)=T+\tilde{\Delta}\left(R, P_{S}\right)
$$

and

$$
\frac{d \tilde{\Delta}}{d R}=\operatorname{sgn} P_{S} \frac{2 E_{+} R^{2}-M^{2}(R) R}{2\left(R-2 E_{+}\right) \sqrt{\mathcal{P}(R)}}-\operatorname{sgn} P_{S} \frac{2 E_{+} R+M^{2}(R)}{2 \sqrt{\mathcal{P}(R)}} .
$$

In the case of dust, $M(R)=$ const, the differential equation (86) reduces to

$$
\frac{d \tilde{\Delta}}{d R}=\operatorname{sgn} P_{S} \frac{2 E_{+}^{2}-M^{2}}{\sqrt{\mathcal{P}(R)}}+\operatorname{sgn} P_{S} \frac{E_{+}\left(4 E_{+}^{2}-M^{2}\right)}{\left(R-2 E_{+}\right) \sqrt{\mathcal{P}(R)}}
$$


where

$$
\mathcal{P}(R):=\left(E_{+}^{2}-M^{2}\right) R^{2}+M^{2} E_{+} R+M^{4} / 4 .
$$

The integration of Eq. (87) is completely analogous to that of (61) and the results are also analogous, only the divergence of $\Delta_{2}$ at $R=0$ is shifted to $R=2 E_{+}$. This divergence of $\tilde{\Delta}_{2}$ at $R=2 E_{+}$does not lead to any problem; it originates from the singularity of the coordinate $T_{+}$on the Kruskal spacetime $\mathcal{M}_{+}$rather that from any geometrical effect. 


\section{REFERENCES}

[1] K. V. Kuchař in Proc. 4th Canadian Conference on General Relativity and Relativistic Astrophysics. World Scientific, Singapore, 1992.

[2] C. J. Isham in Integrable Systems, Quantum Groups, and Quantum Field Theories. Kluver Academic Publishers, London, 1993.

[3] B. S.DeWitt in Dynamical Theory of Groups and Fields. Gordon and Breach, New York, 1965.

[4] M. B. Green, J. H. Schwarz, and E. Witten, Superstrig theory. Cambridge, Cambridge University Press, 1988.

[5] J. F. Donoghue, Helv. Phys. Acta 69 (1996) 269.

[6] P. Hájíček, Commun. Math. Phys. 150 (1992) 545.

[7] P. Hájíček, B. S. Kay, and K. V. Kuchař, Phys. Rev. D46 (1992) 5439.

[8] P. Hájíček in: Canonical Gravity: From Classical to Quantum. Ed. by J. Ehlers and H. Friedrich. Springer, Berlin 1994. P. 137.

[9] P. Hájíček and J. Bičák, Phys. Rev. D56 (1997) 4706.

[10] P. Hájíček and J. Kijowski, Phys. Rev. D57 (1998) 914.

[11] P. Hájíček, Phys. Rev. D57 (1998) 936.

[12] W. Israel, Nuovo Cimento 44B (1966) 1.

[13] K. V. Kuchař, Phys. Rev. D50 (1994) 3961.

[14] J. Kijowski and W. M. Tulczyjev, A Symplectic Framework for Field Theories. Lecture notes in Physics Vol.107, Springer, Berlin, 1979.

[15] J.-M. Souriau, Structure des systèmes dynamiques. Paris, Dunod, 1970. 
[16] P. Hájíček, Nucl. Phys. B (Proc. Suppl.) 57 (1997) 115. 